\title{
Simulation results for an interacting pair of resistively shunted Josephson junctions
}

\author{
Philipp Werner $^{1}$, Gil Refael ${ }^{2,3}$ and Matthias Troyer $^{1}$ \\ ${ }^{1}$ Institut für Theoretische Physik, ETH Hönggerberg, CH-8093 Zürich, \\ Switzerland \\ ${ }^{2}$ Kavli Institute of Theoretical Physics, University of California, \\ Santa Barbara, CA 93106, USA \\ ${ }^{3}$ Department of Physics, California Institute of Technology, Pasadena, \\ CA 91125, USA \\ E-mail: werner@itp.phys.ethz.ch, refael@its.caltech.edu and \\ troyer@itp.phys.ethz.ch
}

Received 11 August 2005

Accepted 2 November 2005

Published 5 December 2005

Online at stacks.iop.org/JSTAT/2005/P12003

doi:10.1088/1742-5468/2005/12/P12003

\begin{abstract}
Using a new cluster Monte Carlo algorithm, we study the phase diagram and critical properties of an interacting pair of resistively shunted Josephson junctions. This system models tunnelling between two electrodes through a small superconducting grain, and is described by a double sine-Gordon model. In accordance with theoretical predictions, we observe three different phases and crossover effects arising from an intermediate coupling fixed point. On the superconductor-to-metal phase boundary, the observed critical behaviour is within error-bars the same as in a single junction, with identical values of the critical resistance and a correlation function exponent which depends only on the strength of the Josephson coupling. We explain these critical properties on the basis of a renormalization group ( $\mathrm{RG}$ ) calculation. In addition, we propose an alternative new mean-field theory for this transition, which correctly predicts the location of the phase boundary at intermediate Josephson coupling strength.
\end{abstract}

Keywords: dissipative systems (theory), quantum Monte Carlo simulations, quantum phase transitions (theory)

ArXiv ePrint: cond-mat/0508163 


\section{Contents}

1. Introduction 2

2. Theory for the symmetric two-junction system 5

2.1. Effective action . . . . . . . . . . . . . . . . 6

2.2. Weak coupling limit . . . . . . . . . . . . . . . . . 6

2.3. Strong coupling limit . . . . . . . . . . . . . . . . . . . . 8

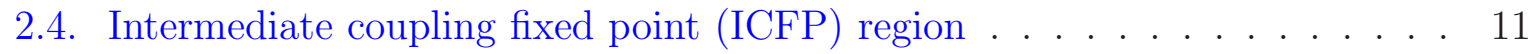

3. Monte Carlo method 13

4. Phase diagram $\quad 15$

4.1. Simulation results . . . . . . . . . . . . . . . . . . . . 15

4.2. Crossover effects in the Monte Carlo results . . . . . . . . . . . . . . 20

5. The critical two-junction system in the ICFP region - comparison with a single Josephson junction 23

5.1. Numerical results and resemblance to the single junction . . . . . . . . . 23

5.2. RG analysis of the critical effective resistance in the two-junction system . 27

6. The ICFP as a self-consistent fixed point 29

7. Correlation function in the NOR phase

8. Conclusions $\quad 36$

$\begin{array}{ll}\text { Acknowledgments } & 37\end{array}$

Appendix A: Critical exponents of the ICFP $\quad 38$

A.1 Weak coupling . . . . . . . . . . . . . . . . . . 38

A.2 Strong coupling regime . . . . . . . . . . . . . . . 39

Appendix B: Approximate calculation of the NOR-FSC phase boundary $\quad 40$

Appendix C: Solution of the circuit in figure $21 \quad 41$

$\begin{array}{ll}\text { References } & 41\end{array}$

\section{Introduction}

The effects of dissipation and decoherence are ubiquitous in quantum systems and influence the properties of materials and nanoscale devices in a profound way. Already the simplest model system, an Ising spin in a transverse field which is coupled to an ohmic heat bath, displays interesting behaviour such as a dynamical phase transition to a localized state at a critical value of the dissipation strength [2]. Another prominent example is the resistively shunted Josephson junction, which undergoes a superconductor-to-metal transition at a critical value of the shunt resistance [3]-[6], which equals the quantum of resistance $R_{\mathrm{Q}}=h / 4 e^{2}=6.5 \mathrm{k} \Omega$. Arrays of Josephson junctions with dissipation have been studied both as a model for granular superconducting films or nanowires [7]-[9], 

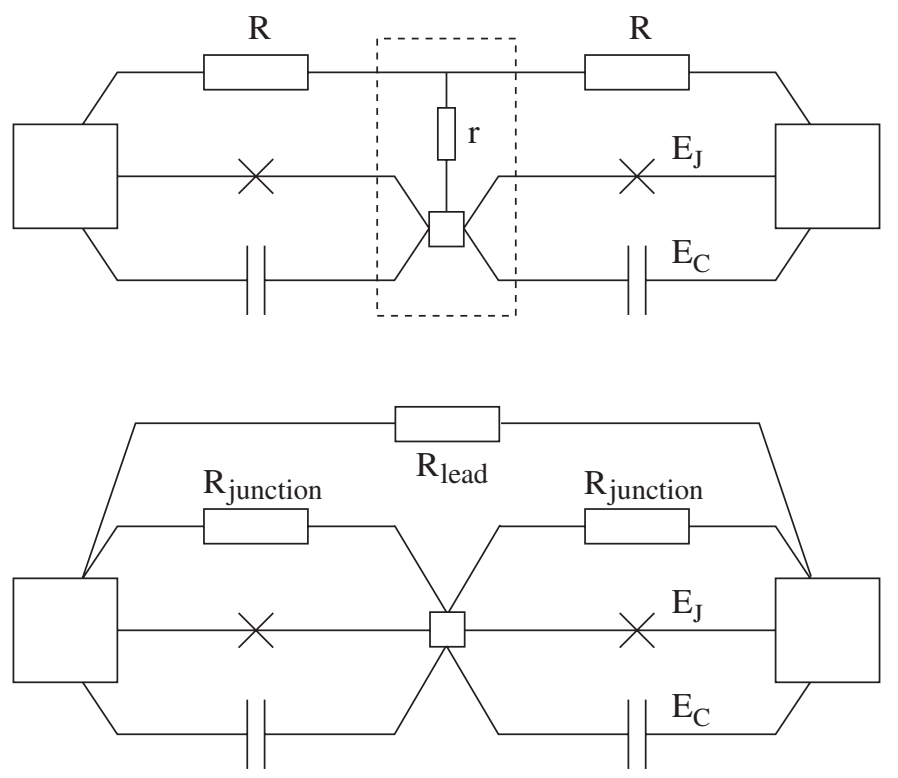

Figure 1. Upper figure: two-junction model considered in [1] with identical shunt resistors $R$ for the left and right junction. The dotted box represents the central grain which incorporates a phenomenological charge relaxation mechanism described by the resistance $r$. Lower figure: equivalent model with modified shunt resistors $R_{\text {junction }}=R+2 r$ and an additional resistor $R_{\text {lead }}=(R / r)(R+2 r)$ connecting the leads.

and in their own right $[10,11]$. The behaviour of these systems, and in particular their superconductor-to-metal phase transition, is far from being completely understood.

Recently, Refael et al have studied a model of a mesoscopic superconducting grain connected to two leads via Josephson tunnelling and shunt resistors [1]. The phase diagram for this simple system was shown to be remarkably complex, with three distinct phases. In addition, contrary to the case of a single resistively shunted Josephson junction, one of the phase boundaries is controlled in part by an intermediate coupling fixed point, and the superconductor-to-normal transition across this boundary can be tuned by the Josephson energy itself. The above effects indicate that although the system discussed in [1] is zero dimensional, it nearly has the full complexity of a one-dimensional array of Josephson junctions. Much of the recent results on Josephson junction chains in [12] draw directly from the two-junction system. The renormalization group $(R G)$ flow equations of the two-junction system are also nearly identical to those of the two-dimensional triangular lattice presented in [13]. In this closely related work on Josephson junction arrays, the local nature of the phase transition, as well as floating phases, have been discussed.

The development of a powerful new cluster Monte Carlo algorithm [14] has allowed us to test and verify several analytical predictions for the single junction and to observe continuously varying correlation exponents along the phase boundary. In this paper we will use adapted versions of these cluster moves to simulate the two-junction model of [1], which is the simplest extension of the single-junction case which exhibits interesting new physics. The model (with identical shunt resistors) is shown in the upper part of figure 1. It consists of two Josephson junctions with coupling energy $E_{\mathrm{J}}$, each shunted with an ohmic 
Simulation results for an interacting pair of resistively shunted Josephson junctions

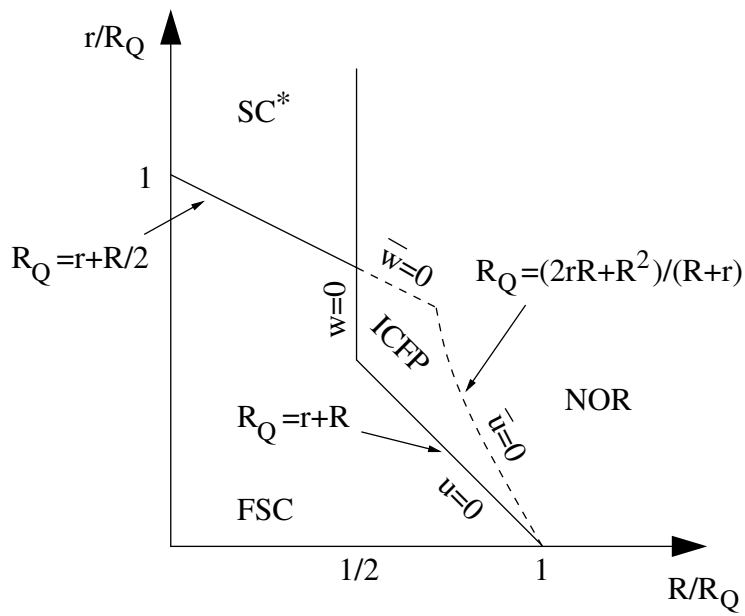

Figure 2. Phase diagram in the limits $E_{\mathrm{J}} / E_{\mathrm{C}} \ll 1$ (solid lines) and $E_{\mathrm{J}} / E_{\mathrm{C}} \gg 1$ (solid and dashed lines). Besides the superconducting (FSC) and metallic (NOR) phase, the two-junction system can be in a state which is superconducting from lead to lead, although the individual junctions are insulating $\left(\mathrm{SC}^{*}\right)$. The parameters $u, w$ and $\bar{u}, \bar{w}$ are defined in equations (22) and (26). The values $u=0$ and $w=0$ define the FSC-NOR boundary in the weak coupling limit, and $\bar{u}=0$ and $\bar{w}=0$ in the strong coupling limit.

resistor $R$. On the central grain, the model incorporates a 'charge relaxation mechanism' (supposed to represent the break-up of Cooper pairs into electrons) which is described by an additional resistor $r$.

Dissipation produced by electrons flowing through the resistors reduces phase fluctuations between the superconducting islands which they connect. This can be seen from the dissipative action term in equation (4), and it explains how strong dissipation leads to superconducting phase coherence. Depending on the values of $R$ and $r$, three different phases occur.

- The individual junctions are insulating and there is no supercurrent from lead to lead: normal phase (NOR).

- The junctions are superconducting and thus also the whole device from lead to lead: fully superconducting phase (FSC).

- The individual junctions are insulating but there is superconducting phase coherence from lead to lead: $\mathrm{SC}^{*}$ phase.

The phase diagram for the limiting cases $E_{\mathrm{J}} \ll E_{\mathrm{C}}$ and $E_{\mathrm{J}} \gg E_{\mathrm{C}}$ has been computed in [1] using an RG approach and is shown in figure 2 as a function of the resistances $R$ and $r$. The boundary between the NOR- and FSC-phase in the region marked ICFP depends on the value of $E_{\mathrm{J}} / E_{\mathrm{C}}$ as well as the values of the resistors $r$ and $R$. The behaviour of the system in this region is controlled by an intermediate coupling fixed point (ICFP), which will be discussed in section 2.4.

In the remains of this paper we present the results of a thorough Monte Carlo (MC) investigation of the two-junction system. Each result is compared with predictions or explanations based on the RG flow equations presented in section 2. After discussing 
the various phases of the two-junction system, we explain in section 3 the Monte Carlo method which was used to investigate the model numerically. In particular, we discuss several types of efficient cluster updates, which are adaptations of the recently developed rejection-free cluster algorithm for single resistively shunted junctions [14].

In section 4 we identify the three phases NOR, FSC and $\mathrm{SC}^{*}$ by computing the temperature dependence of the lead-to-lead and lead-to-grain resistance. The numerically obtained phase diagram at intermediate Josephson coupling is compared to the theoretical predictions for weak and strong Josephson coupling. There is a good agreement between theory and simulation results, except in the region where the three phases meet. We explain these small deviations of the measured phase boundaries from the predicted ones in terms of slow crossovers in the RG flow, which prevent the MC simulation from exploring the zero-temperature behaviour.

In section 5 we concentrate on the critical FSC-NOR line in the ICFP region. In particular, we compare the critical properties of the two-junction system to those of a single junction with the same Josephson coupling $E_{\mathrm{J}} / E_{\mathrm{C}}$, and a shunt resistance $R_{\mathrm{s}}=R_{\mathrm{Q}}$. The two systems exhibit (within error-bars) identical behaviour in their correlation functions, fluctuations and the effective resistance of the critical junctions. These critical properties depend on the value of $E_{\mathrm{J}} / E_{\mathrm{C}}$ but not on the value of the resistors $r$ and $R$. In section 5.2, we try to explain this rather surprising observation in terms of the effective junction resistances of the two-junction system, calculated from the $\mathrm{RG}$ flow of section 2 . We show that the predicted critical resistance exhibits only a weak dependence on the (shunt) resistors and agrees quite well with the measured value.

In section 6, however, we pursue a different path to explain the remarkable resemblance between the single resistively shunted junction and the two-junction system. We show that the data can be well explained by a 'mean-field' theory, which treats one of the two junctions as an effective resistor. This is a new way of approaching the double sine-Gordon model action (equation (1) below). The assumption is that, on the FSCNOR phase boundary, each junction sees an environment which imitates a shunt resistor $R_{\mathrm{S}}=R_{\mathrm{Q}}$ and that it may be replaced by a resistor whose value can be found from the critical resistance of the single resistively shunted junction (with the same Josephson coupling). On the basis of these assumptions it is possible to derive an expression for the position of the FSC-NOR phase boundary, which fits the MC data and the RG-based predictions quite well.

In section 6 we consider the phase-phase correlation functions in the NOR phase within the ICFP region. For fixed resistors, we measure a strong dependence of the correlation exponents on the Josephson coupling strength, which is in contrast to the single-junction model, where these exponents depend only on the value of the shunt resistor. This behaviour is explained as a consequence of the flow in the additional Josephson coupling $J_{+}$between the leads, which is generated under the RG and not present in the single-junction model.

\section{Theory for the symmetric two-junction system}

In this section we will present the effective action for the symmetric two-junction system, and then briefly discuss its RG flow equations and the various phases. This discussion will prove to be especially useful when interpreting the Monte Carlo results. 
We will first describe the NOR-SC* and $\mathrm{SC}^{*}-\mathrm{FSC}$ transitions in the weak and strong coupling limits. It is important to note that the $\mathrm{SC}^{*}$ phase appears due to interactions between the two junctions, and can not be understood in terms of the physics of a single junction. Finally, we will discuss the important intermediate coupling fixed point which controls the direct NOR-FSC transition.

\subsection{Effective action}

The imaginary-time effective action of the symmetric two-junction system can be written as a functional of the phase differences $\phi_{1}$ and $\phi_{2}$ across the first and second junction,

$$
S_{\text {eff }}\left[\phi_{1}, \phi_{2}\right]=S_{\mathrm{C}}\left[\phi_{1}, \phi_{2}\right]+S_{\mathrm{J}}\left[\phi_{1}, \phi_{2}\right]+S_{\mathrm{D}}\left[\phi_{1}, \phi_{2}\right],
$$

where the charging term $S_{\mathrm{C}}$, the Josephson coupling term $S_{\mathrm{J}}$ and the dissipation term $S_{\mathrm{D}}$ read

$$
\begin{aligned}
S_{\mathrm{C}}\left[\phi_{1}, \phi_{2}\right]= & \frac{1}{16 E_{\mathrm{C}}} \int_{0}^{\beta} \mathrm{d} \tau\left[\left(\frac{\mathrm{d} \phi_{1}}{\mathrm{~d} \tau}\right)^{2}+\left(\frac{\mathrm{d} \phi_{2}}{\mathrm{~d} \tau}\right)^{2}\right], \\
S_{\mathrm{J}}\left[\phi_{1}, \phi_{2}\right]= & -E_{\mathrm{J}} \int_{0}^{\beta} \mathrm{d} \tau\left[\cos \left(\phi_{1}\right)+\cos \left(\phi_{2}\right)\right], \\
S_{\mathrm{D}}\left[\phi_{1}, \phi_{2}\right]= & \frac{R_{\mathrm{Q}}}{R(R+2 r)} \int_{0}^{\beta} \mathrm{d} \tau \mathrm{d} \tau^{\prime} \frac{(\pi / \beta)^{2}}{\sin ^{2}\left((\pi / \beta)\left(\tau-\tau^{\prime}\right)\right)} \\
& \times\left[R\left(\phi_{1}(\tau)-\phi_{1}\left(\tau^{\prime}\right)\right)^{2}+R\left(\phi_{2}(\tau)-\phi_{2}\left(\tau^{\prime}\right)\right)^{2}\right. \\
& \left.+r\left(\left(\phi_{1}(\tau)+\phi_{2}(\tau)\right)-\left(\phi_{1}\left(\tau^{\prime}\right)+\phi_{2}\left(\tau^{\prime}\right)\right)\right)^{2}\right] .
\end{aligned}
$$

$E_{\mathrm{C}}=e^{2} / 2 C$ is the (single-electron) charging energy of each junction and sets the overall energy scale. $E_{\mathrm{J}}$ denotes the coupling strengths of the junctions. Ohmic dissipation in the resistors is introduced using the model of Caldeira and Leggett $[15,16]$.

The system discussed in [1] is equivalent to the one illustrated in the lower part of figure 1 , where each junction is shunted by a resistor

$$
R_{\text {junction }}=R+2 r
$$

and the leads are connected by an additional resistor

$$
R_{\text {lead }}=(R / r)(R+2 r) \text {. }
$$

This is a consequence of the ' $Y-\Delta^{\prime}$ ' transformation of resistor networks [1]. For simplicity, we consider a capacitive coupling between the leads and central grain only and no Josephson coupling between the leads. Such a coupling will be generated by the RG flow.

\subsection{Weak coupling limit}

When the Josephson coupling energy $E_{\mathrm{J}}$ is small, it can be used as a small parameter for a perturbative RG analysis. As explained in [1], in addition to the bare Josephson energy of the two junctions, the RG flow produces yet another Josephson coupling - the co-tunnelling $J_{+}$. In the same sense that $E_{\mathrm{J}}$ is the amplitude for a pair-hopping between the leads and the grain, $J_{+}$is the amplitude for a Cooper pair to tunnel between the two 


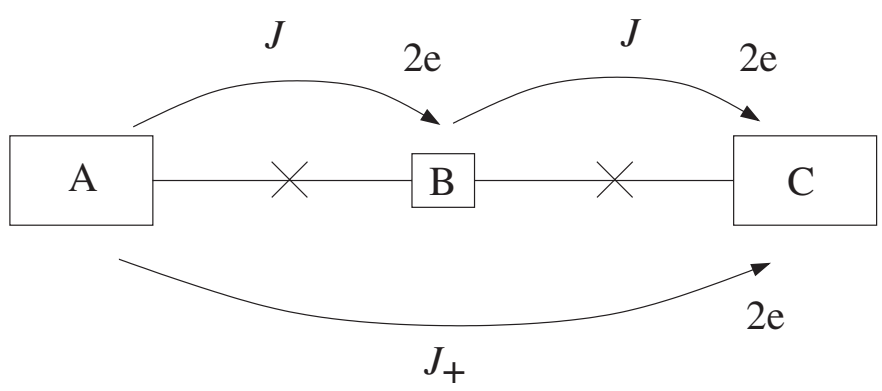

Figure 3. In the weak coupling limit, we consider the RG flow of the Josephson couplings $J$ (with $J_{0}=E_{\mathrm{J}}$ ) and $J_{+} . J$ is the amplitude for Cooper-pair hopping between either of the leads and the grain. $J_{+}$is the amplitude for Cooper-pair tunnelling from lead to lead. This process is generated in the second order of the RG flow.

leads, skipping over the grain (see figure 3). To be more specific, the Cooper-pair hopping conductivities are

$$
G_{\mathrm{AB}}=G_{\mathrm{BC}} \sim E_{\mathrm{J}}^{2}, \quad G_{\mathrm{AC}} \sim J_{+}^{2} .
$$

Let us quote here the RG equations for the Josephson strengths in the symmetric case [1]. To distinguish between the bare Josephson energy $E_{\mathrm{J}}$ and the renormalized one, we use $J$ to denote the flow of $E_{\mathrm{J}}$. For the Josephson strengths $J$ of the junction and $J_{+}$ between the leads we get

$$
\begin{aligned}
& \frac{\mathrm{d} J}{\mathrm{~d} l}=J\left(1-\frac{R+r}{R_{\mathrm{Q}}}\right)+\frac{R}{R_{\mathrm{Q}}} J J_{+}, \\
& \frac{\mathrm{d} J_{+}}{\mathrm{d} l}=J_{+}\left(1-\frac{2 R}{R_{\mathrm{Q}}}\right)+\frac{r}{R_{\mathrm{Q}}} J^{2} .
\end{aligned}
$$

The Josephson coupling $J_{+}$between the leads is originally zero, but will be generated under the flow in the second order in $J$. From these RG equations we can infer the scaling behaviour of the Cooper-pair conductivities in the asymptotic low-temperature regime and in the ICFP area. This is done by identifying the temperature $T$ with the RG scale as follows:

$$
T \sim \mathrm{e}^{-l}
$$

The normal phase of the system is described by the fixed point $J=J_{+}=0$, in which the Josephson junctions are insulating (see figure 4). The Josephson coupling in this phase (and by equation (7) thus also the Cooper-pair conductivities) are expected to vanish as a power law in $T$,

$$
J \sim T^{-\left(1-\left(R+r / R_{\mathrm{Q}}\right)\right)}, \quad J_{+} \sim T^{-\left(1-\left(2 R / R_{\mathrm{Q}}\right)\right)} .
$$

The signature of the normal phase is a drop of both lead-to-lead and lead-to-island conductance as the temperature is reduced.

If $J_{+}$is relevant at first order $\left(R<R_{\mathrm{Q}} / 2\right)$, then at low temperatures points $\mathrm{A}$ and $\mathrm{C}$ in figure 3 become short circuited, so the leads become phase coherent independently of $J$. The RG equation for $J$ becomes

$$
\frac{\mathrm{d} J}{\mathrm{~d} l}=J\left(1-\frac{r+R / 2}{R_{\mathrm{Q}}}\right) .
$$




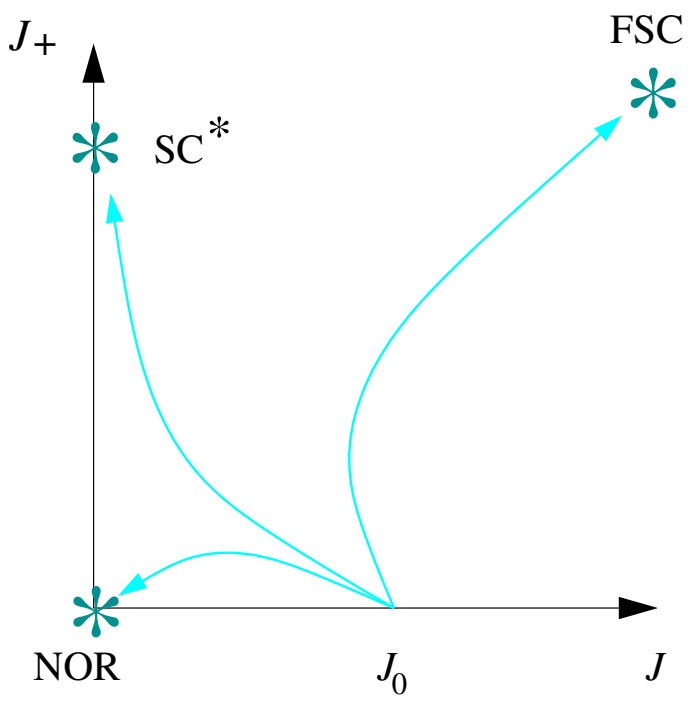

Figure 4. Depending on the values of the resistors $r$ and $R$, the low-energy behaviour of the two-junction system is described by three stable fixed points. In terms of the weak coupling Josephson variables $J$ and $J_{+}$, these points are as follows. (i) Normal (NOR) fixed point at $J=J_{+}=0$, where Josephson tunnelling is suppressed. (ii) Fully superconducting (FSC) point in which both $J$ and $J_{+}$are large. (iii) $\mathrm{SC}^{*}$ point where $J_{+}$is large and $J=0$. Here, Cooper-pair tunnelling is suppressed from lead to grain, but is very strong between the two leads.

When $J$ is irrelevant $\left(r+R / 2>R_{\mathrm{Q}}\right)$, the junctions are insulating despite the phase coherence between the leads. This is the $\mathrm{SC}^{*}$ phase, and it is described by the fixed point shown in figure 4 . The conductivities of the individual junctions in this phase vanish as the square of $J$, which follows the power law

$$
J \sim T^{\left((r+R / 2) / R_{\mathrm{Q}}\right)-1} .
$$

The lead-to-lead conductance diverges as a power law of the temperature and this divergence will be discussed in the next section using the strong coupling analysis. Note that the signature of the $\mathrm{SC}^{*}$ phase is an increase in the lead-to-lead conductance, and a drop in the lead-to-island conductance as the temperature decreases.

The FSC phase occurs when both $J$ and $J_{+}$are relevant $\left(r+R / 2<R_{\mathrm{Q}}\right)$. The weak coupling equations describe the initial increase in the Josephson couplings. The divergence of the conductance, however, is described in the next section, where the strong coupling limit is discussed.

\subsection{Strong coupling limit}

When $E_{\mathrm{J}}$ is large, one can no longer treat the Josephson coupling as a perturbation. But by employing a duality, one can describe the system in terms of phase slips [1]. Phase slips describe a sudden winding of a phase difference across a junction. There are two kinds of phase slip we need to consider: individual phase slips and phase-slip dipoles. Individual phase slips create a potential drop, and hence dissipation in a junction independently of 
a.

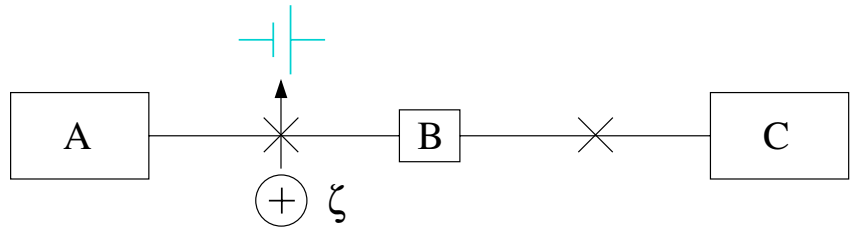

b.

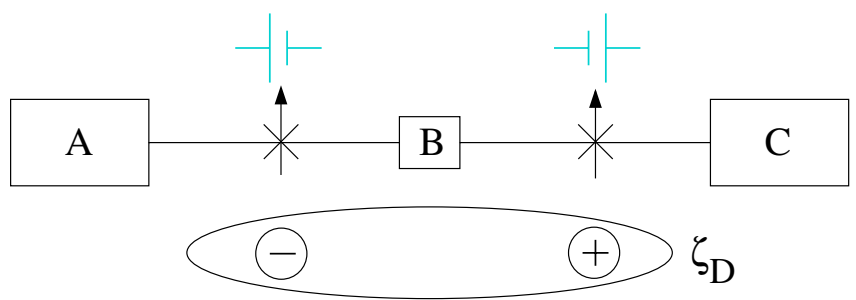

Figure 5. In the strong coupling limit we consider the RG flow of the phase slip fugacities $\zeta$ and $\zeta_{\mathrm{D}}$. (a) $\zeta$ is the amplitude, or fugacity, for a single phase slip across either of the junctions that connect the leads to the middle grain. When a phase slip occurs it produces a momentary potential drop across the junction, which leads to dissipation. (b) When $r>0$, interactions between phase slips in the two junctions produce a phase-slip dipole in second order in the RG. The fugacity of the dipoles is $\zeta_{\mathrm{D}}$. When a dipole crosses the system there are two simultaneous momentary voltage drops with opposite directions on the two junctions, such that there is no potential drop between the leads, A and C.

the other junction; we denote their fugacity by $\zeta$. A phase slip in junction AB is partially screened if an anti-phase slip (a slip with the opposite winding) simultaneously occurs in junction BC. This partial screening leads to the generation of phase-slip dipoles in the $\mathrm{RG}$ flow, in a very similar fashion to the generation of $J_{+}$. We denote the dipole fugacity by $\zeta_{\mathrm{D}}$. By determining how often phase slips occur, $\zeta$ and $\zeta_{\mathrm{D}}$ determine the effective resistance across the Josephson junctions (see figure 5):

$$
R_{\mathrm{AB}}=R_{\mathrm{BC}} \sim \zeta^{2}+\zeta_{\mathrm{D}}^{2}, \quad R_{\mathrm{AC}} \sim 2 \zeta^{2} .
$$

The phase-slip dipoles do not affect the lead-to-lead resistance since a dipole produces a voltage blip in one junction and an opposite voltage blip in the other junction. Hence, the two blips add up to zero, and do not produce a voltage drop between the leads.

The flow equations for $\zeta$ and $\zeta_{\mathrm{D}}$ are

$$
\begin{aligned}
& \frac{\mathrm{d} \zeta}{\mathrm{d} l}=\zeta\left(1-\frac{R_{\mathrm{Q}}(R+r)}{2 R r+R^{2}}\right)+\frac{R R_{\mathrm{Q}}}{R^{2}+2 R r} \zeta \zeta_{\mathrm{D}}, \\
& \frac{\mathrm{d} \zeta_{\mathrm{D}}}{\mathrm{d} l}=\zeta_{\mathrm{D}}\left(1-\frac{2 R R_{\mathrm{Q}}}{R^{2}+2 R r}\right)+\frac{r R_{\mathrm{Q}}}{R^{2}+2 R r} \zeta^{2} .
\end{aligned}
$$

In the FSC phase both $\zeta$ and $\zeta_{\mathrm{D}}$ are irrelevant, and decay to zero as a power law in temperature (and so do the resistances by equation (14)),

$$
\zeta \sim T^{-\left(1-\left(R_{\mathrm{Q}}(R+r) /\left(2 R r+R^{2}\right)\right)\right)}, \quad \zeta_{\mathrm{D}} \sim T^{-\left(1-\left(2 R R_{\mathrm{Q}} /\left(R^{2}+2 R r\right)\right)\right)} .
$$




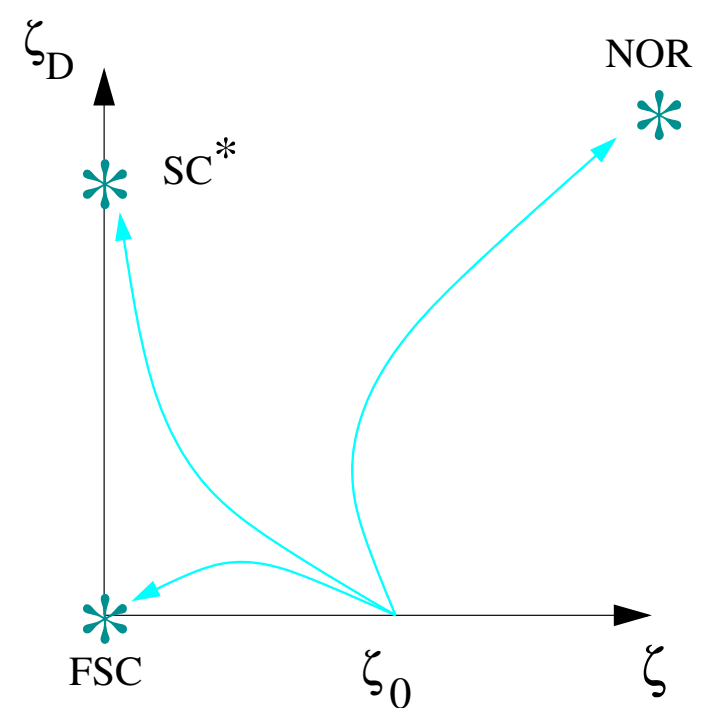

Figure 6. In terms of the strong coupling phase-slip fugacities $\zeta$ and $\zeta_{\mathrm{D}}$, the three stable fixed points are the following. (i) Fully superconducting (FSC) fixed point at $\zeta=\zeta_{\mathrm{D}}=0$. In this point both single phase slips and phase-slip dipoles are suppressed. (ii) Normal (NOR) point in which both $\zeta$ and $\zeta_{\mathrm{D}}$ are large. There is no phase coherence and Cooper-pair tunnelling in the system, which corresponds to weak coupling. (iii) The $\mathrm{SC}^{*}$ point where $\zeta_{\mathrm{D}}$ is large and $\zeta=0$. Phase coherence is maintained between the leads (points $\mathrm{A}$ and $\mathrm{C}$ ) but there is no phase coherence between the leads and the grain B. A quick look at figure 4 reveals the strong-weak duality of this system.

The signature of the superconducting phase is thus a drop in both the lead-to-lead and lead-to-grain resistance. This phase is controlled by the $\zeta=\zeta_{\mathrm{D}}=0$ fixed point (figure 6). Note that the FSC and NOR phases are very similar in their behaviour to the singlejunction problem which has been investigated numerically in [14].

When $\zeta_{\mathrm{D}}$ becomes relevant $\left(r+R / 2>R_{\mathrm{Q}}\right)$, phase coherence is destroyed between the leads and the central grain, but not between the two leads. At low temperatures, this practically implies that the effective resistance of the resistor $r$ is diverging. Therefore the flow equation for $\zeta$ becomes

$$
\frac{\mathrm{d} \zeta}{\mathrm{d} l}=\zeta\left(1-\frac{R_{\mathrm{Q}}}{2 R}\right) .
$$

When $R<R_{\mathrm{Q}} / 2$, even though $\zeta_{\mathrm{D}}$ has diverged, $\zeta$ remains irrelevant and decays to zero at low temperatures as

$$
\zeta \sim T^{\left(R_{\mathrm{Q}} / 2 R\right)-1} .
$$

In this phase the lead-to-lead resistance falls off as $\zeta^{2}$, but the lead-to-grain resistance diverges as $1 / J^{2}$ from equation (14). Again, this is the $\mathrm{SC}^{*}$ phase, and the corresponding fixed point is shown in figure 6 .

In the normal phase, both $\zeta$ and $\zeta_{\mathrm{D}}$ diverge at low energy scales, and one should use equations (8), (9) and (11) to describe the low-temperature behaviour of the measured resistances. 


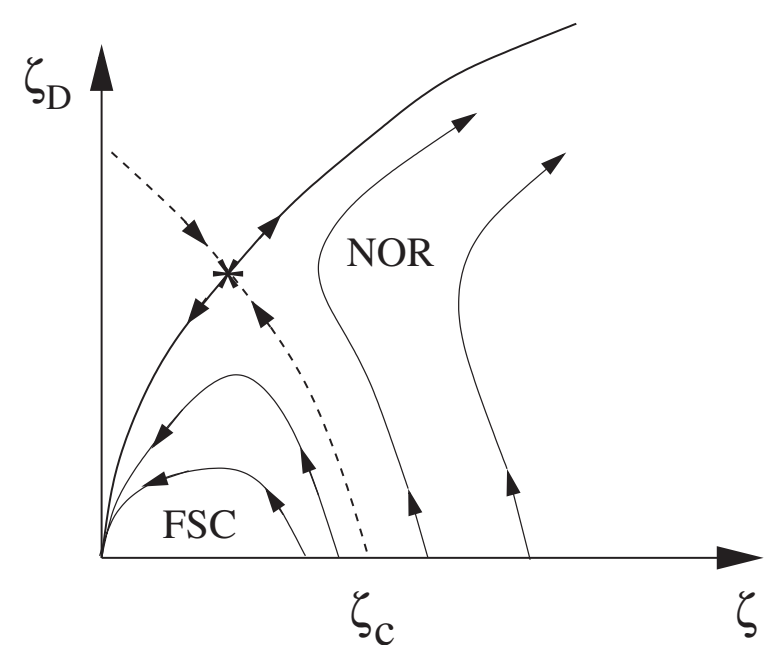

Figure 7. Typical flow of the parameters $\zeta$ and $\zeta_{\mathrm{D}}$. If the initial values of $\zeta$ and $\zeta_{\mathrm{D}}$ are in the region marked FSC they flow towards $\zeta=\zeta_{\mathrm{D}}=0$ after flowing towards the intermediate coupling fixed point. If they are initially in the region marked NOR, they flow towards the weak coupling (NOR) fixed point, again, after flowing to the ICFP first. The dashed line is the critical manifold of the NOR-FSC transition, and the arrows near the fixed point mark the eigendirections of the linearized flow. The physical line is $\zeta_{\mathrm{D}}=0$, and therefore the critical $\zeta_{\mathrm{c}}$ for the NOR-FSC transition is given by the intersection of the critical manifold with the $\zeta_{\mathrm{D}}=0$ axis.

\subsection{Intermediate coupling fixed point (ICFP) region}

In the ICFP region a fourth fixed point appears (in addition to the $\mathrm{FSC}, \mathrm{SC}^{*}$, and NOR fixed points), and the scaling behaviour of $J$ and $J_{+}$, or $\zeta$ and $\zeta_{\mathrm{D}}$, is determined at intermediate temperatures by this fixed point. At lower temperatures the asymptotic $T \rightarrow 0$ scaling behaviour is determined by the $J=J_{+}=0$ (normal phase) fixed point, or by the $\zeta=\zeta_{\mathrm{D}}=0$ one (FSC phase). As opposed to the other regions of the phase diagram, where the resistors alone determine the phase, in the ICFP region, the phase of the system also depends on the value of $E_{\mathrm{J}} / E_{\mathrm{C}}$. These parameters determine the critical value of the initial amplitude of Cooper-pair hopping, or, using the strong coupling picture, of the phase-slip fugacities. In this region there is no $\mathrm{SC}^{*}$ phase, and the transition between the FSC and NOR phases is direct. An illustration of this situation in the ICFP region is given in figure 7 .

The existence of the unstable fixed point is evident from the nonlinear flow equations for the weak and strong coupling limits. We start with the weak coupling flow equations (equations (8) and (9)) written as follows:

$$
\begin{aligned}
& \frac{\mathrm{d} J}{\mathrm{~d} l}=-J u+\frac{R}{R_{\mathrm{Q}}} J J_{+}, \\
& \frac{\mathrm{d} J_{+}}{\mathrm{d} l}=-J_{+} w+\frac{r}{R_{\mathrm{Q}}} J^{2},
\end{aligned}
$$


where

$$
u=\frac{R+r}{R_{\mathrm{Q}}}-1, \quad w=\frac{2 R}{R_{\mathrm{Q}}}-1 .
$$

When both $u>0$ and $w>0$, the RG equations (20) and (21) have a third fixed point (in addition to zero and $\infty$ ). This point is at

$$
J^{*}=\frac{R_{\mathrm{Q}}}{\sqrt{r R}} \sqrt{u w}, \quad J_{+}^{*}=\frac{R_{\mathrm{Q}}}{R} u .
$$

Therefore the lines $u=0$ and $w=0$ mark the weak coupling boundaries of the ICFP region (see figure 2).

Similarly, in the strong coupling limit we can write the flow equations (15) and (16) as

$$
\begin{aligned}
& \frac{\mathrm{d} \zeta}{\mathrm{d} l}=-\zeta \bar{u}+\frac{R R_{\mathrm{Q}}}{R^{2}+2 R r} \zeta \zeta_{\mathrm{D}}, \\
& \frac{\mathrm{d} \zeta_{\mathrm{D}}}{\mathrm{d} l}=-\zeta_{\mathrm{D}} \bar{v}+\frac{r R_{\mathrm{Q}}}{R^{2}+2 R r} \zeta^{2},
\end{aligned}
$$

where

$$
\bar{u}=\frac{R_{\mathrm{Q}}(R+r)}{2 R r+R^{2}}-1, \quad \bar{w}=\frac{2 R R_{\mathrm{Q}}}{R^{2}+2 R r}-1 .
$$

As in the weak coupling limit, when $\bar{u}>0$ and $\bar{w}>0$, an unstable fixed point appears at intermediate values of $\zeta$ and $\zeta_{\mathrm{D}}$. The lines $\bar{u}>0$ and $\bar{w}>0$ mark the boundaries of the ICFP region on the strong coupling side. The fixed-point fugacities of the ICFP are given by

$$
\zeta^{*}=\frac{R^{2}+2 r R}{R_{\mathrm{Q}} \sqrt{r R}} \sqrt{\overline{u w}}, \quad \zeta_{\mathrm{D}}^{*}=\frac{R^{2}+2 r R}{R_{\mathrm{Q}} R} \bar{u} .
$$

The scaling properties of the system near criticality and at intermediate energy scales are determined by the critical properties of the above ICFP and, in particular, the relevant and irrelevant directions and the exponents associated with them: $\lambda_{+}$and $\lambda_{-}$. These properties are calculated in appendix A.

Qualitatively the situation is illustrated in figure 7. Consider a generic flow of $\zeta$ and $\zeta_{\mathrm{D}}$ at some value of the resistors $r$ and $R$. If the system we are considering has initial fugacities $\zeta^{(0)}$ and $\zeta_{\mathrm{D}}^{(0)}$ in the FSC-region, then both $\zeta$ and $\zeta_{\mathrm{D}}$ flow towards 0 eventually, and the system is in the superconducting phase at low energies. But this behaviour is not at all trivial: before decaying to zero, $\zeta_{\mathrm{D}}$ starts off growing as $\zeta$ decreases. Similarly, if $\zeta^{(0)}$ and $\zeta_{\mathrm{D}}^{(0)} \sim 0$ are in the NOR region, once the flow passes by the ICFP, both $\zeta$ and $\zeta_{\mathrm{D}}$ become relevant. But before the flow reaches the fixed point, there is an energy range in which $\zeta_{\mathrm{D}}$ grows, but $\zeta$ decreases. Generally speaking, the extent of this range of energies is determined by the irrelevant critical exponent, $\lambda_{-}$of the ICFP (see appendix A).

The FSC-NOR transition occurs when $\zeta^{(0)}$ and $\zeta_{\mathrm{D}}^{(0)}$ are on the critical manifold which flows exactly into the ICFP. In the two-junction system $\zeta_{\mathrm{D}}^{(0)}=0$, and therefore there is a critical fugacity $\zeta_{\mathrm{c}}$ above which the system is in the NOR phase, and below which it is in the FSC phase. The initial phase slip fugacity is determined by the ratio $E_{\mathrm{J}} / E_{\mathrm{C}}$, 
and therefore this transition (for a given value of the resistances $R$ and $r$ in the ICFP region) can be tuned by changing the Josephson energy. The qualitative picture described above is equally valid in weak coupling, where the only difference would be discussing the pair-tunnelling amplitudes, $J$ and $J_{+}$, rather than the phase-slip fugacities.

The flow of the fugacities (or pair tunnelling amplitudes) before reaching the ICFP determines the behaviour of the resistance as a function of temperature at intermediate temperatures. In these temperature ranges the behaviour of the resistance may be misinterpreted as any of the three phases of the system. Particularly, in the region of parameter space where $\zeta$ decreases and $\zeta_{\mathrm{D}}$ increases, the lead-to-lead resistance decreases, since it is only proportional to $\zeta^{2}$, but the lead-to-grain resistance increases. This behaviour could be misinterpreted as the system being in the $\mathrm{SC}^{*}$ phase. In order to determine the true $T=0$ phase one has to investigate the system at very low temperatures. These crossover effects indeed appear explicitly in the Monte Carlo simulations of the system.

\section{Monte Carlo method}

Monte Carlo simulations of the two-junction model as shown in the lower part of figure 1 can be performed using variants of the local and cluster updates detailed in [14]. Imaginary time is divided into $N$ time slices of size $\Delta \tau=\beta / N$ and the variables $\phi_{1, n}$ and $\phi_{2, n}$, $n=1, \ldots, N$ (phase differences across the junctions at the discrete times $\tau_{n}=n \Delta \tau$ ) are used to represent the phase configuration (see figure 8). We implemented the following types of Monte Carlo updates.

(1) Single-junction cluster updates

A cluster of connected sites is constructed in one of the junctions as outlined in [14]. The cost in action of flipping the cluster, $\Delta S_{\text {lead }}$, which is associated with the last term in equation (4),

$$
\begin{aligned}
S_{\text {lead }}=\frac{R_{\mathrm{Q}}}{R_{\text {lead }}} \int_{0}^{\beta} \mathrm{d} \tau \mathrm{d} \tau^{\prime} \frac{(\pi / \beta)^{2}}{\sin ^{2}\left((\pi / \beta)\left(\tau-\tau^{\prime}\right)\right)} \\
\quad \times\left(\left(\phi_{1}(\tau)+\phi_{2}(\tau)\right)-\left(\phi_{1}\left(\tau^{\prime}\right)+\phi_{2}\left(\tau^{\prime}\right)\right)\right)^{2},
\end{aligned}
$$

must be calculated and the cluster move accepted with probability

$$
p=\min \left(1, \exp \left(-\Delta S_{\text {lead }}\right)\right) \text {. }
$$

(2) Single-junction local updates

Local updates in Fourier space are proposed in one of the junction, as detailed in [14]. They, too, are accepted with probability

$$
p=\min \left(1, \exp \left(-\Delta S_{\text {lead }}\right)\right) .
$$

(3) Compensated single-junction cluster updates

The $\mathrm{SC}^{*}$-phase is characterized by phase coherence between the leads $\left(\phi_{1}(\tau)+\right.$ $\phi_{2}(\tau) \approx$ constant) but strong fluctuations of the variables $\phi_{1}(\tau)$ and $\phi_{2}(\tau)$ (insulating junctions). Therefore, the fluctuations in the two junctions essentially compensate each other and efficient updates in the $\mathrm{SC}^{*}$-phase should take this constraint into account. 

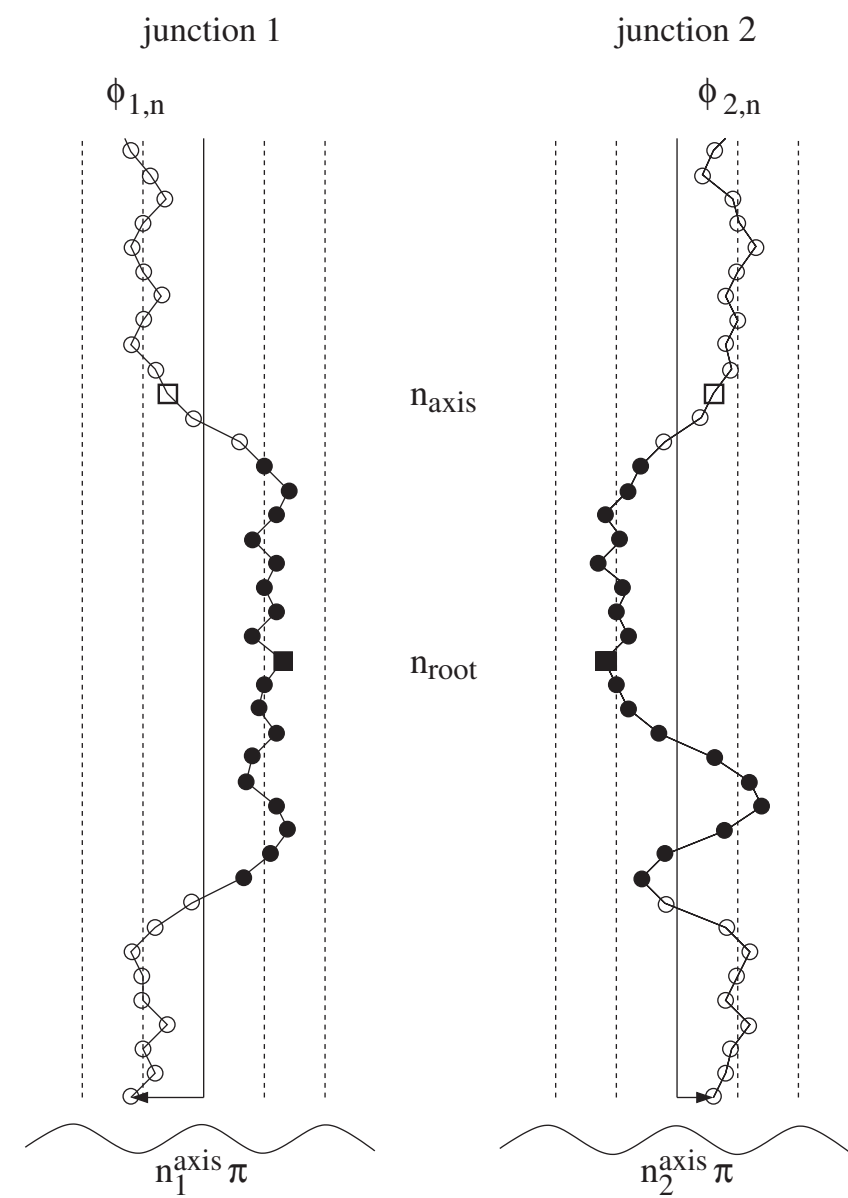

Figure 8. Illustration of the two-junction cluster updates. The axis is chosen at a symmetry point of the potential neighbouring $\phi_{i, n_{\text {axis }}}\left(n_{\text {axis }}\right.$ is a random site), but on opposite sides in the junction $i=1$ and 2. A second random site $n_{\text {root }}$ is picked as the root site of the cluster. The sites connected to the root site are determined using the bond probabilities (33) and are marked with black dots. The new configuration is obtained by flipping the cluster around the axis. In other words, the spin-like variables defined relative to the axis and shown as an arrow on the first site are inverted during a cluster update.

In a compensated single-junction cluster update, a cluster of connected sites is constructed in one of the junctions as outlined in [14] and an equal, but opposite, move proposed for each phase variable in the other junction. Since $\Delta \phi_{1}+\Delta \phi_{2}=0$, there is no cost in action associated with the $S_{\text {lead }}$-term in equation (4). However, there is a cost in action, $\Delta S_{\mathrm{oj}}$, associated with the contribution from the other junction (hence the notation 'oj'),

$$
\begin{aligned}
S_{\mathrm{oj}}=\frac{1}{16 E_{\mathrm{C}}} \int_{0}^{\beta} \mathrm{d} \tau\left(\frac{\mathrm{d} \phi_{\mathrm{oj}}}{\mathrm{d} \tau}\right)^{2}-E_{\mathrm{J}} \int_{0}^{\beta} \mathrm{d} \tau \cos \left(\phi_{\mathrm{oj}}\right) \\
+\frac{R_{\mathrm{Q}}}{R_{\text {junction }}} \int_{0}^{\beta} \mathrm{d} \tau \mathrm{d} \tau^{\prime} \frac{(\pi / \beta)^{2}\left(\phi_{\mathrm{oj}}(\tau)-\phi_{\mathrm{oj}}\left(\tau^{\prime}\right)\right)^{2}}{\sin ^{2}\left((\pi / \beta)\left(\tau-\tau^{\prime}\right)\right)} .
\end{aligned}
$$


The compensated cluster move should therefore be accepted with probability

$$
p=\min \left(1, \exp \left(-\Delta S_{\mathrm{oj}}\right)\right) \text {. }
$$

(4) Two-junction cluster updates

A random site $k$ is picked and an axis $n_{i}$ in each of the two junctions chosen among the two closest to $\phi_{i}\left(\tau_{k}\right)$, such that $n_{1} \pi \leq \phi_{1, k}$ and $n_{2} \pi \geq \phi_{2, k}$ or vice versa (see figure 8). Relative coordinates $\phi_{i}^{\text {axis }}=\phi_{i}-n_{i}^{\text {axis }} \pi$ are introduced in both junctions and a cluster of sites connected to a randomly chosen root site is constructed using the bond probabilities

$$
p(k, l)=\max \left(0,1-\exp \left(-\Delta S_{k, l}\right)\right)
$$

where the cost in action of breaking a bond, $\Delta S_{k, l}$, is defined as

$$
\begin{aligned}
\Delta S_{k, l} & =\sum_{i=1,2}\left(S\left(\phi_{i, k}^{\text {axis }},-\phi_{i, l}^{\text {axis }}\right)-S\left(\phi_{i, k}^{\text {axis }}, \phi_{i, l}^{\text {axis }}\right)\right) \\
& =8 g(k-l) \sum_{i=1,2} \phi_{i, k}^{\text {axis }} \phi_{i, l}^{\text {axis }} .
\end{aligned}
$$

In the above expression, $g(j)$ is the kernel $(j \neq 0)$

$$
g(j)=\frac{1}{32 E_{\mathrm{C}} \Delta \tau}\left(\delta_{j, 1}+\delta_{j, N-1}\right)+\frac{1}{8 \pi^{2}} \frac{R_{\mathrm{Q}}}{R_{\text {junction }}} \frac{(\pi / N)^{2}}{\sin ((\pi / N) j)^{2}} .
$$

Hence, in contrast to the single-junction case, a cluster can contain relative phase variables of both signs. The cluster building process (34) takes into account the capacitive, dissipative and Josephson contributions from both junctions, but not the dissipative contribution from the $S_{\text {lead }}$-term. A two-junction cluster move therefore can only be accepted with probability

$$
p=\min \left(1, \exp \left(-\Delta S_{\text {lead }}\right)\right) \text {. }
$$

\section{Phase diagram}

We first use the efficient Monte Carlo scheme outlined in section 3 to identify the three phases NOR, FSC and $\mathrm{SC}^{*}$, and to determine the phase diagram for an intermediate value of the Josephson coupling $E_{\mathrm{J}}$. This allows us to test the theoretical predictions outlined in section 2 and in figure 2.

The result of this study is shown in figure 11 and explained in section 4.1. The agreement between the Monte Carlo calculation and the theory is very good. Small deviations, however, appear in the vicinity of the (tricritical) meeting point of the three phases: $r=0.75 R_{\mathrm{Q}}$ and $R=R_{\mathrm{Q}} / 2$. These deviations from the theoretically predicted phase diagram are explained in section 4.2 as crossover effects, and are indirect evidence for the existence of the intermediate coupling fixed point.

\subsection{Simulation results}

We use the resistance at imaginary frequencies to identify the state of conductance between the leads and from the leads to the central grain. The imaginary frequency resistance is 
Simulation results for an interacting pair of resistively shunted Josephson junctions
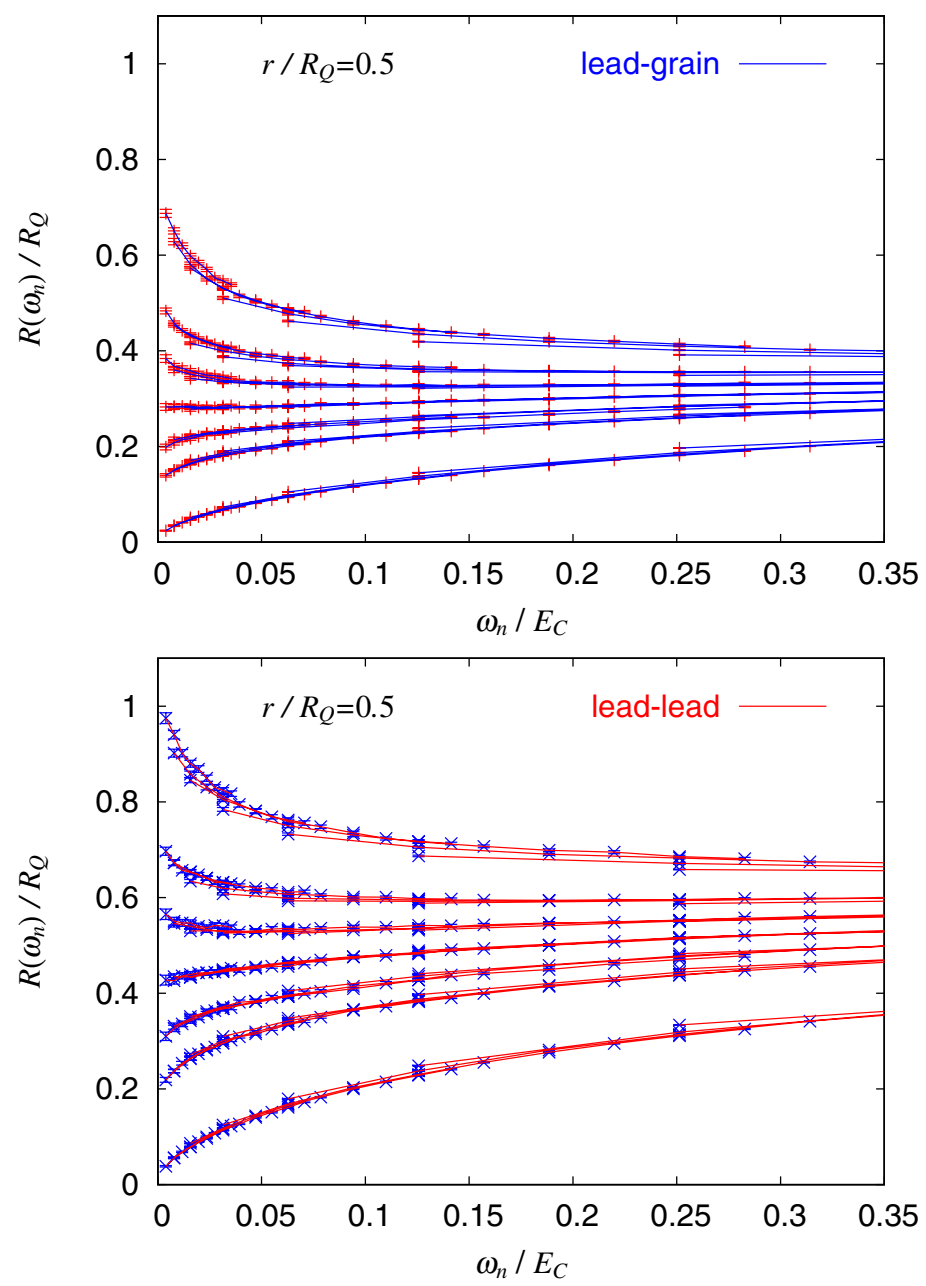

Figure 9. Imaginary frequency resistances for $r=0.5$ and $\beta E_{\mathrm{C}}=25,50,100$, 200, 400, 800 and 1600. Only the first ten Matsubara points are shown for each temperature and the extrapolation of these curves to zero frequency gives the zero-bias resistance. From bottom to top, the different sets of curves correspond to $R=0.5,0.6,0.625,0.65,0.675,0.7$ and 0.75 . The curves in the upper panel show the resistance from lead to central grain, those in the lower panel the resistance from lead to lead. In both cases the superconductor-to-normal phase transition occurs at $R \approx 0.65$.

defined as

$$
\frac{R\left(\omega_{n}\right)}{R_{\mathrm{Q}}}=\frac{1}{2 \pi}\left|\omega_{n}\right|\langle\phi \phi\rangle_{\omega_{n}}
$$

where $\omega_{n}=(2 \pi n) / \beta$ denotes a Matsubara frequency and $\langle\phi \phi\rangle_{\omega_{n}}$ the Fourier transform of the phase-phase correlation function $\langle\phi(0) \phi(\tau)\rangle\left(\phi \equiv \phi_{i}\right.$ in the case of conductance from lead to central grain and $\phi \equiv \phi_{1}+\phi_{2}$ for the conductance from lead to lead).

In figures 9 and 10 we show the results of such an analysis obtained for $E_{\mathrm{J}} / E_{\mathrm{C}}=1$ and $\Delta \tau E_{\mathrm{C}}=0.25$. The interpretation of the data is the same as in the single-junction case discussed in [14]. Figure 9 shows the transition across the FSC-NOR phase boundary 
Simulation results for an interacting pair of resistively shunted Josephson junctions
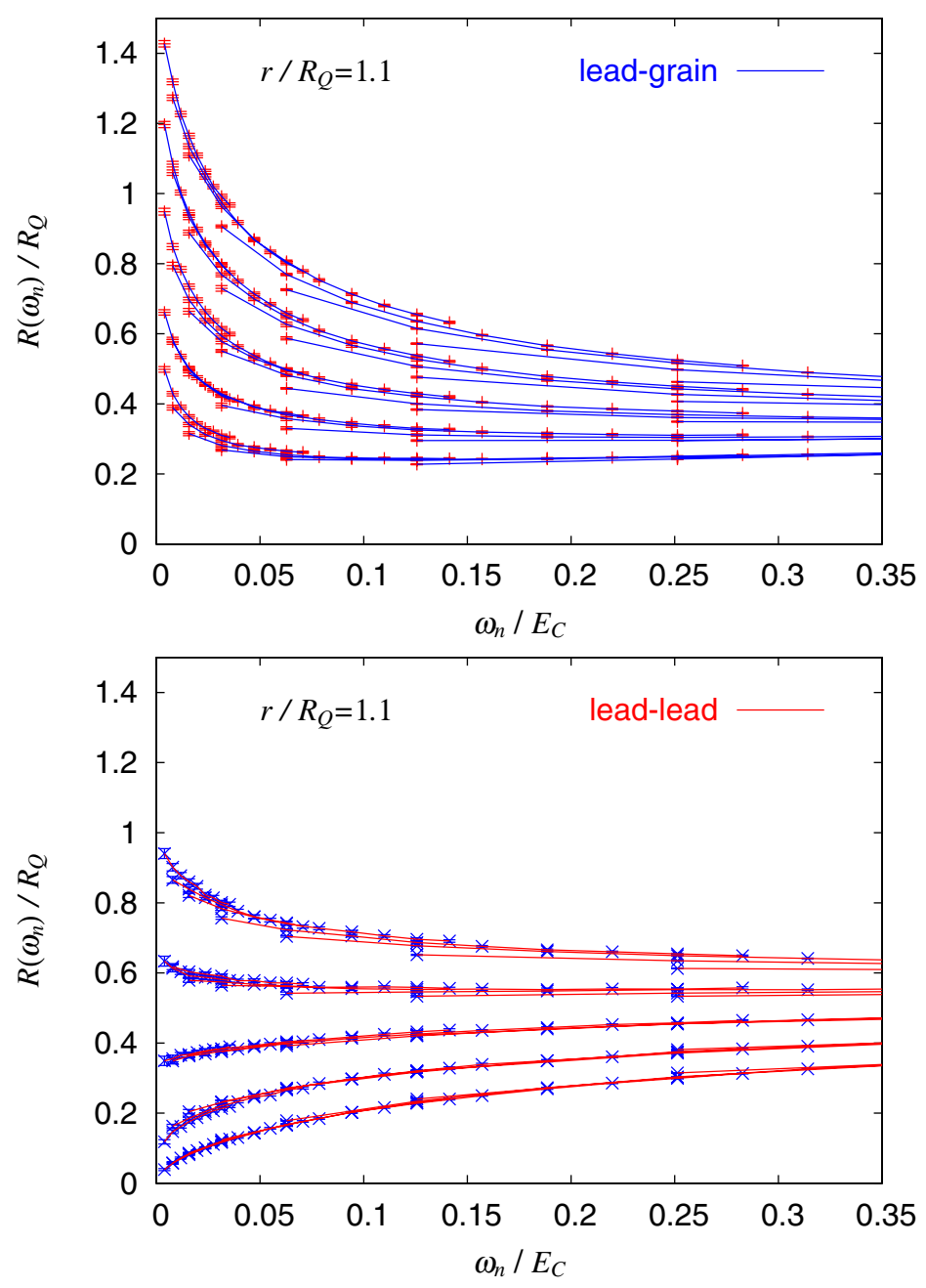

Figure 10. Imaginary frequency resistances for $r=1.1$ and $\beta E_{\mathrm{C}}=25,50,100$, 200, 400, 800 and 1600. Only the first ten Matsubara points are shown for each temperature and the extrapolation of these curves to zero frequency gives the zero-bias resistance. From bottom to top, the different sets of curves correspond to $R=0.4,0.45,0.5,0.55$ and 0.6 . The curves in the upper panel show the resistance from lead to central grain, those in the lower panel the resistance from lead to lead. For $R<0.5$, the junctions are insulating, but the device from lead to lead is superconducting ( $\mathrm{SC}^{*}$ phase).

at $r=0.5 R_{\mathrm{Q}}$. The different sets of curves correspond to different values of $R$ and the different lines in each set of curves to different temperatures. Only the lowest ten Matsubara frequencies are shown. Extrapolating these curves to $\omega_{n} \rightarrow 0$ yields the zero bias resistance, which decreases to zero with decreasing temperature in the $T=0$ superconducting state. If the junction turns insulating with decreasing temperature, the resistance increases and eventually saturates at the value $r+R$ (from lead to central grain) or $2 R$ (from lead to lead). It is obvious from the data in figure 9 that the transition occurs simultaneously in the individual junctions and between the leads, in accordance with theoretical predictions. 
Simulation results for an interacting pair of resistively shunted Josephson junctions

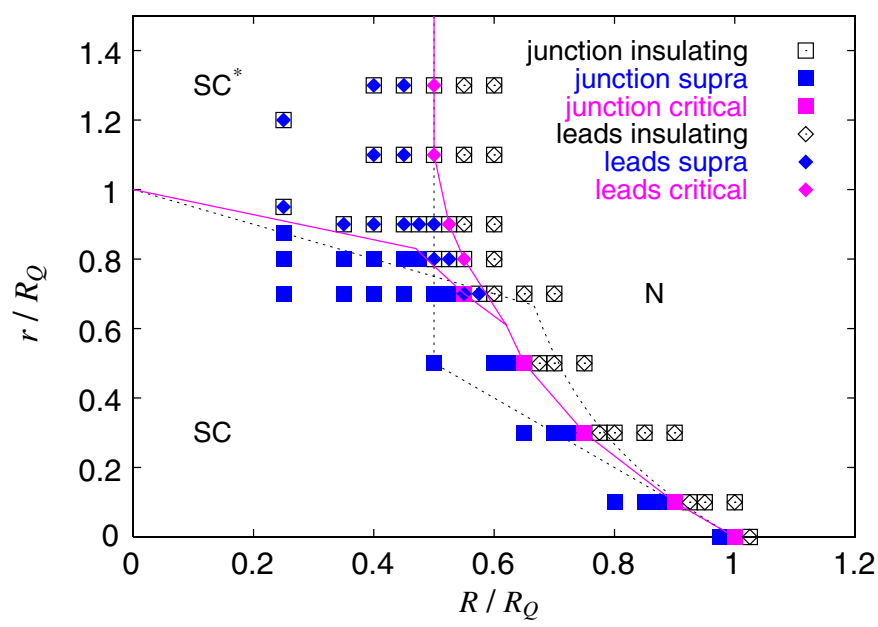

Figure 11. Phase diagram obtained from the analysis of the resistance-versustemperature behaviour. We find a good agreement with theoretical predictions (black dotted lines), except in the region around the tricritical point, where the three phases meet.

Figure 10 shows the transition across the $\mathrm{SC}^{*}-\mathrm{NOR}$ phase boundary at $r=1.1$. First of all, we note that the data for $R<0.5 R_{\mathrm{Q}}$ clearly prove the existence of the $\mathrm{SC}^{*}$ phase. While the individual junctions turn insulating as $T \rightarrow 0$, the resistance from lead to lead decreases to zero as the temperature decreases $(T=0$ superconductivity). If $R$ is increased, however, the device undergoes a (lead-to-lead) superconductor-to-metal transition at $R=0.5 R_{\mathrm{Q}}$, as predicted by theory.

Repeating this type of analysis for several values of $r$ we could map out the phase diagram which is shown for $E_{\mathrm{J}} / E_{\mathrm{C}}=1$ in figure 11. As expected, the FSC-NOR phase boundary lies somewhere in between the limiting values calculated analytically for $E_{\mathrm{J}} / E_{\mathrm{C}} \gg 1$ and $E_{\mathrm{J}} / E_{\mathrm{C}} \ll 1$, indicated by the dotted lines (see also figure 2). A closeup of the FSC-NOR phase boundary is shown in figure 12; the measured critical line at $E_{\mathrm{J}} / E_{\mathrm{C}}=1$ agrees very well with an RG-based calculation (presented in appendix B).

Overall, we find a good agreement with the theoretically predicted phase diagram, except in the vicinity of the tricritical point, $r=0.75 R_{\mathrm{Q}}, R=R_{\mathrm{Q}} / 2$, where the three phases meet. Phase boundaries there appear to be shifted to a somewhat larger value of $R$ and a smaller value of $r$. As it turns out, in this region, crossover effects are important and determining the phase from finite-temperature simulations can be misleading. A detailed discussion of the observed deviations is given below, in section 4.2.

As the Josephson coupling strength $E_{\mathrm{J}}$ is increased from zero to infinity, the FSCNOR phase boundary shifts from the weak coupling to the strong coupling limit indicated in figure 2. This behaviour is demonstrated in figure 13, which shows a cut across the critical surface at $r / R_{\mathrm{Q}}=0.5$.

Since the dc resistance is defined as a limit $\omega \rightarrow 0$ on the real frequency axis, one may legitimately ask whether our procedure of extrapolating along the imaginary frequency axis is safe. The method will not work if $R(\omega)$ develops a singularity on or near the real axis in the range $|\omega|<T$. In the case of a single junction, such an emergence of a new energy scale can be ruled out [5]. Also in the two-junction model, the only two energy scales are $E_{\mathrm{J}}$ 
Simulation results for an interacting pair of resistively shunted Josephson junctions

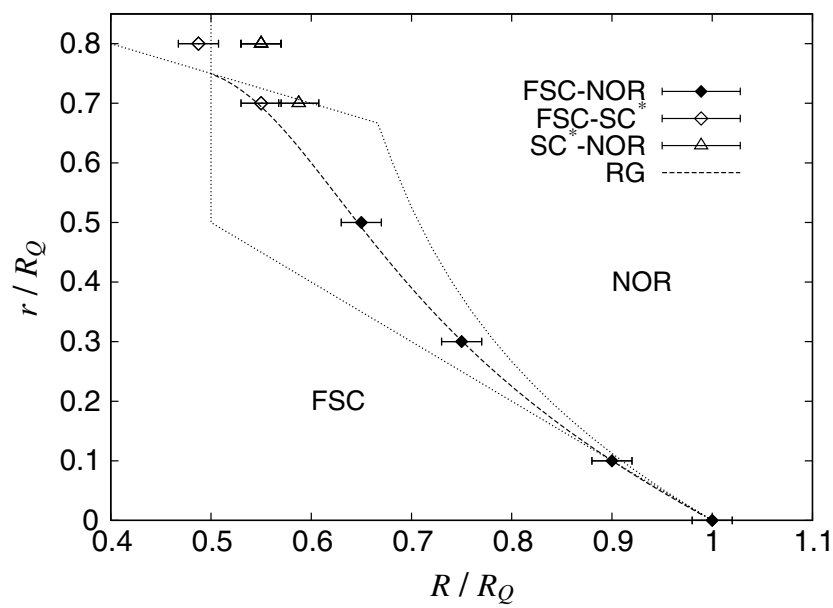

Figure 12. A close-up of the phase diagram in the ICFP region for $E_{\mathrm{J}} / E_{\mathrm{C}}=1$. The dashed line is the RG prediction for the FSC-NOR phase boundary as obtained in appendix B, with the bare phase slip fugacity $\zeta_{0}=0.295$ (the only fitting parameter) determined from the critical point at $r=R_{\mathrm{Q}} / 2$. The RG results agree well with the Monte Carlo results. Filled dots mark the FSC-NOR phase boundary and empty dots an $\mathrm{FSC}-\mathrm{SC}^{*}$ or $\mathrm{SC}^{*}-\mathrm{FSC}$ transition.

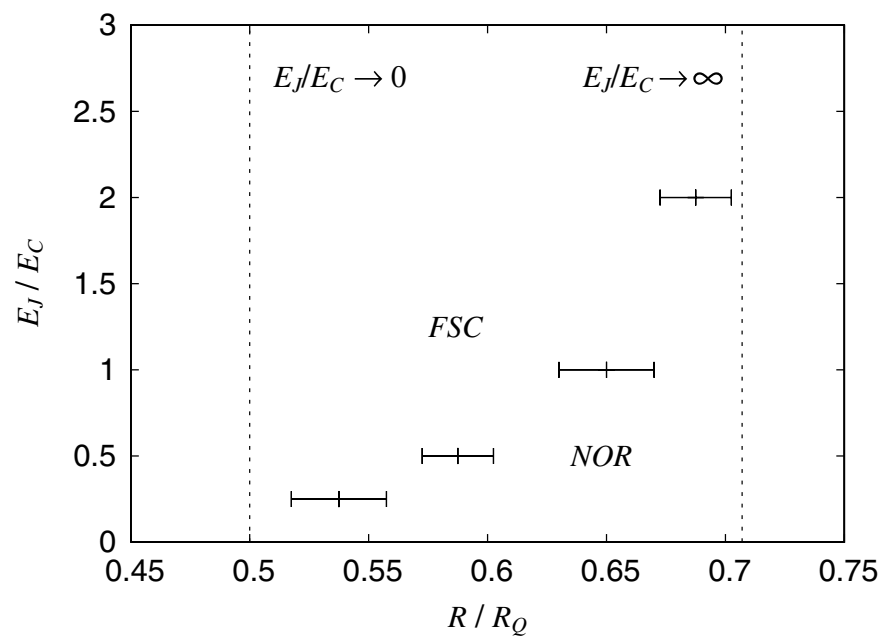

Figure 13. Phase boundary as a function of the Josephson coupling strength calculated at $r / R_{\mathrm{Q}}=0.5$. The dashed lines indicate the analytically predicted values for the critical $R$ in the weak and strong coupling limits.

and $E_{\mathrm{C}}$ and the temperatures reached by our simulations are far below these scales. For the single-junction data reported in [14] we have checked that higher-order Padé approximants $([2 / 0],[2 / 1], \ldots,[3 / 3])$ produce no disturbing poles. While the extrapolated resistance values away from criticality depend somewhat on the fitting function, the qualitative features (resistance increasing/decreasing with temperature) do not. Since we only use the latter robust property to locate the phase transition line and to determine the critical resistance, we believe that our estimates are reliable. 
Of course, any extrapolation of numerical data will fail if a crossover occurs at an inaccessibly low temperature. The implications of this for the interpretation of figure 11 will be discussed in the following section.

\subsection{Crossover effects in the Monte Carlo results}

The theory of the two-junction system as outlined in section 2 and in appendix A leads us to expect crossover behaviour at intermediate temperatures. Although the range of temperatures at our disposal is limited, and the crossover regime spans a narrow range of energy scales, we see indirect evidence of the crossover effects in the measured phase diagram. As mentioned above, near the meeting point of the three phases, the predicted phase boundaries do not completely agree with the Monte Carlo simulation results (figure 11). We will explain these deviations using the RG analysis.

The deviations of the measured data from the theoretical predictions can be understood by looking at the RG trajectories given by equations (15) and (16). As explained in section 2.4, the flow of the phase slip fugacities or pair-tunnelling amplitudes near the intermediate coupling fixed point can make the measured resistance as a function of temperature behave as though the system is in the $\mathrm{SC}^{*}$ phase. Near the meeting point of the three phases, the flow towards the ICFP is extremely slow; it is dominated by the critical exponent $\lambda_{-}$given by equations (A.9) and (A.22):

$$
\begin{aligned}
& \lambda_{-}^{\text {(weak) }}=\frac{1}{2}\left(-w-\sqrt{w^{2}+8 u w}\right), \\
& \lambda_{-}^{\text {(strong) }}=\frac{1}{2}\left(-\bar{w}-\sqrt{\bar{w}^{2}+8 \overline{u w}}\right),
\end{aligned}
$$

where $u, w$ and $\bar{u}, \bar{w}$ are defined in equations (22) and (26). More specifically, in this regime $x-x^{*} \sim T^{\lambda_{-}}$with $x$ being $\zeta, \zeta_{\mathrm{D}}$, or $J, J_{+}$, and $x^{*}$ the respective fixed-point value. As can be seen from equations (38), (39) and figure $2, \lambda_{-}$in both the weak and strong coupling regimes vanishes at the meeting point $(w=\bar{w}=0)$, and therefore is expected to be small near this special point for any value of the Josephson coupling. This implies that since the Monte Carlo calculation is limited to temperatures above $T=E_{c} / 2500$ (if we choose $\Delta \tau E_{\mathrm{C}}=0.25$, which seems appropriate) it may not probe the ground state of the system, but rather the crossover physics. This will lead to distorted NOR-FSC phase boundaries in this region. As it turns out, this phenomenon is not restricted to the ICFP region: slow crossovers occur all around the triple point and may shift the observed NOR-SC* and $\mathrm{SC}^{*}-\mathrm{FSC}$ phase boundaries, as indeed is seen in figure 11.

To demonstrate the above behaviour we plot the predicted RG flow of the phase slip fugacities in the strong coupling limit for four values of $r / R_{\mathrm{Q}}$ in the region of interest (figures 14 and 15). We colour coded the plot according to the RG flow parameter $l=\ln \Lambda / T$ where $\Lambda$ is the ultraviolet cutoff. Roughly speaking, the maximum RG scale we probe in the Monte Carlo calculation is $l_{\max } \approx 7$, and therefore we stop the colour coding at the value $l=10$. The various flow lines all start with the same initial fugacity (same $E_{\mathrm{J}} / E_{\mathrm{C}}$ ), but have the resistance $R$ varied across the transition. In the Monte Carlo simulation one measures the lead-to-lead resistance, which we expect to behave as

$$
R_{\mathrm{AC}} \sim \zeta^{2}
$$


a.

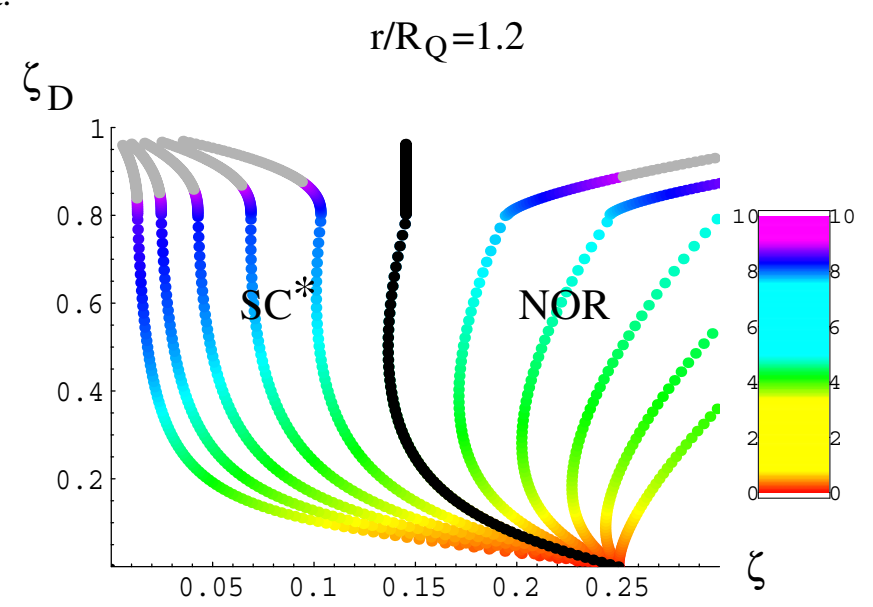

b.

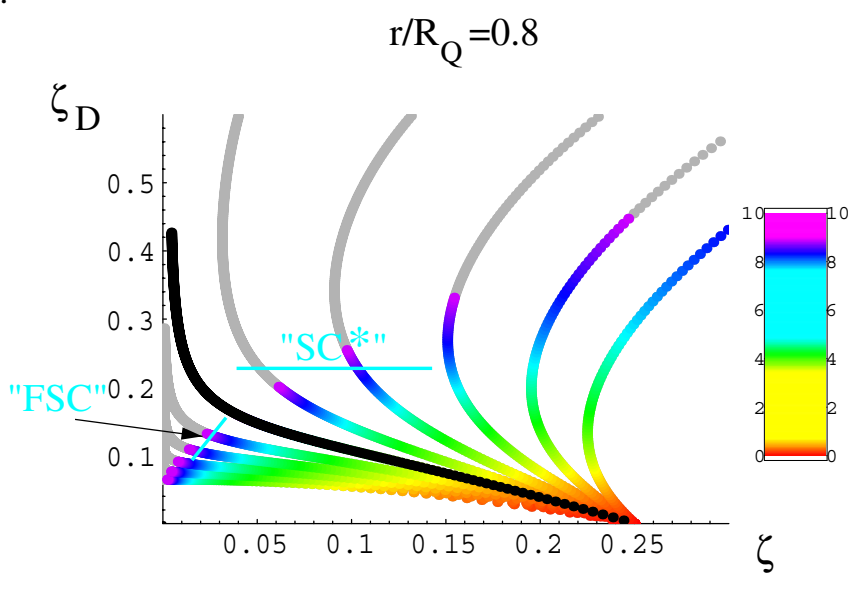

Figure 14. Simulated RG flows of phase slip fugacities (equations (15) and (16)). The initial conditions for all plots are $\zeta^{(0)}=0.25, \zeta_{\mathrm{D}}^{(0)}=0$. The colour coding marks the RG flow parameter $l=\ln \Lambda / T$, and regions not accessible with Monte Carlo simulations $(l>10)$ are shown in light grey. (a) $r / R_{\mathrm{Q}}=1.2, R / R_{\mathrm{Q}}$ varies from 0.4 to 0.6 from left to right. The black curve is at the critical value $R=R_{\mathrm{Q}} / 2$. Within the energy scales probed by the Monte Carlo simulation it is easy to distinguish between the $\mathrm{SC}^{*}$ and NOR phase. In the $\mathrm{SC}^{*}$ phase $\zeta$ decreases making the lead-to-lead resistance $R_{\mathrm{AC}} \sim \zeta^{2}$ decrease at low energies, while $\zeta_{\mathrm{D}}$ grows rapidly, producing an insulating regime for the effective resistance for each junction, $R_{\mathrm{AB}}$. We assume that $\zeta_{\mathrm{D}}$ begins to saturate at $\zeta_{\mathrm{D}}=0.7$ and makes the flow cross over to equation (18). (b) $r / R_{\mathrm{Q}}=0.8$, and $R / R_{\mathrm{Q}}$ varies as in (a). At this value of $r$ the temperature range accessible with simulations is no longer sufficient to determine the true phase boundaries. For a range of $R$-values near and above the critical point $R=R_{\mathrm{Q}} / 2$ we may mistakenly identify the phase as $\mathrm{SC}^{*}$, since $R_{\mathrm{AC}} \sim \zeta^{2}$ is decreasing, but $R_{\mathrm{AB}} \sim \zeta^{2}+\zeta_{\mathrm{D}}^{2}$ is increasing. Similarly, for $R<R_{\mathrm{Q}} / 2$ it looks as if the system is in the FSC phase (instead of the $\mathrm{SC}^{*}$ phase), since $\zeta_{\mathrm{D}}$ grows very slowly. This explains the deviations of the numerically determined phase boundaries (figure 11) from the theoretical ones. 
c.

$$
\mathrm{r} / \mathrm{R}_{\mathrm{Q}}=0.7
$$

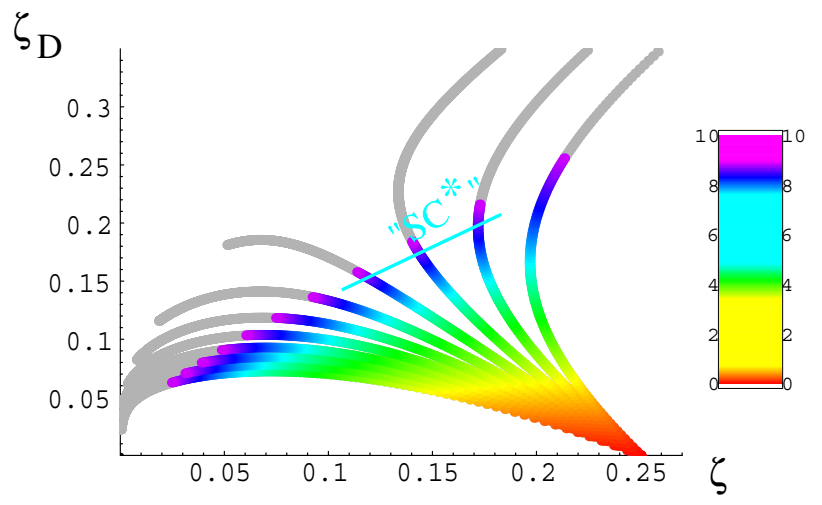

d.

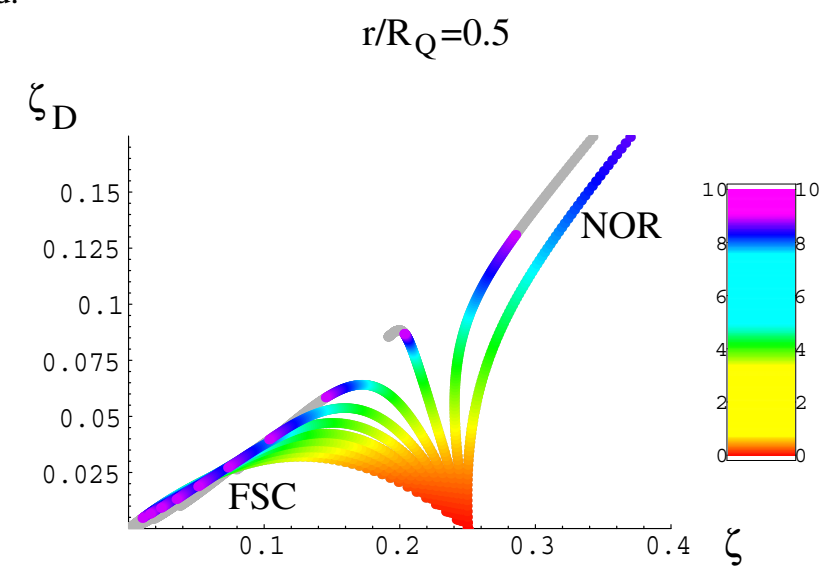

Figure 15. Same as figure 14. (c) $r / R_{\mathrm{Q}}=0.7, R / R_{\mathrm{Q}}$ varies between 0.5 and 0.6 from left to right. These flows are in the ICFP region. Even though in this region we expect a direct NOR-FSC transition, at the energy range accessible in the Monte Carlo simulation we again see a region which would be mistaken as $\mathrm{SC}^{*}$, which is indeed the case in figure 11. The slow crossover is due to the proximity to the triple point at $r=0.75 R_{\mathrm{Q}}$ where the three phases meet. (d) $r / R_{\mathrm{Q}}=0.5$, and $R / R_{\mathrm{Q}}$ varies as in (c). As in figure 14(a) even within the energy range accessible numerically, we clearly see the NOR and FSC phases showing up $-\zeta$ and $\zeta_{\mathrm{D}}$ either both diverge or both decrease at the lowest temperatures accessible by Monte Carlo simulation.

and the lead-to-grain resistance, which goes as

$$
R_{\mathrm{AB}}=R_{\mathrm{BC}} \sim \zeta^{2}+\zeta_{\mathrm{D}}^{2}
$$

As can be seen from figure 14(a), at large values of $r, r / R_{\mathrm{Q}}=1.2$, the flow within the accessible range of $l$ allows an easy determination of the phase: at low energies, $\zeta$ slowly decays or diverges to the left or the right of the black critical flow line at $R=R_{\mathrm{Q}} / 2$, while $\zeta_{\mathrm{D}}$ strongly diverges (for this illustration we assumed that when $\zeta_{\mathrm{D}}>0.7$ the grain starts becoming effectively insulating). In figure $14(\mathrm{~b})$, at $r / R_{\mathrm{Q}}=0.8$ closer to the meeting point (which is at $r / R_{\mathrm{Q}}=0.75$ ) we see that this strong distinction cannot be made. There is a whole region of parameters to the right of the black line (marking the theoretically 
predicted critical flow), in which the lead-to-lead resistance $\left(R_{\mathrm{AC}} \sim \zeta^{2}\right)$ decreases, and the single-junction resistance $\left(R_{\mathrm{AB}} \sim \zeta^{2}+\zeta_{\mathrm{D}}^{2}\right)$ increases. This region eventually flows to the normal fixed point, but in the finite-temperature Monte Carlo simulations this cannot be observed and it appears that an $\mathrm{SC}^{*}$ phase exists at values $R>R_{\mathrm{Q}} / 2$, as shown in figure 11. To the left of the black line in figure $14(\mathrm{~b})$ we theoretically expect the $\mathrm{SC}^{*}$ phase, but even there we see a crossover which will be misinterpreted as an FSC phase: $R_{\mathrm{AB}}$ decreases and, for a range of parameters, also $R_{\mathrm{AC}}$. $R_{\mathrm{AC}}$ only starts to grow at a lower energy scale, disclosing the true $\mathrm{SC}^{*}$ phase. Indeed, in figure 11 we see that the Monte Carlo calculation indicates an FSC region at $r / R_{\mathrm{Q}}=0.8$ and $R<0.5 R_{\mathrm{Q}}$, where it should be the $\mathrm{SC}^{*}$ phase.

In the ICFP region we again encounter slow crossovers associated with the intermediate coupling fixed point. As can be seen in figure $15(\mathrm{c})$ with $r / R_{\mathrm{Q}}=0.7$, there is a range of parameters which would be mistaken for the $\mathrm{SC}^{*}$ phase, in which $R_{\mathrm{AC}}$ seems to drop, while $R_{\mathrm{AB}}$ grows. Since the $R G$ is stopped approximately at the $l_{\max }$ corresponding to our Monte Carlo calculation, the observed flow in this case is dominated only by the critical exponent $\lambda_{-}$of equations (38) and (39), which vanishes at the triple point. For comparison, in figure $15(\mathrm{~d})$ we show the RG flow for $r / R_{\mathrm{Q}}=0.5$. In this case the RG does flow to the stable NOR and FSC fixed points at energy scales higher than the lowest temperature accessible in our calculation. Indeed, in this parameter range there is no longer any evidence of an $\mathrm{SC}^{*}$-like phase in the Monte Carlo calculation.

\section{The critical two-junction system in the ICFP region-comparison with a single Josephson junction}

In this section we investigate the direct NOR-FSC transition, and compare the critical behaviour of the two-junction system with that of a single junction with the same $E_{\mathrm{J}} / E_{\mathrm{C}}$. We make a rather surprising observation: along this phase boundary, the effective resistance of the junction, the temperature dependence of the mean phase fluctuations, and the correlation exponents are within error-bars the same as those in a single resistively shunted Josephson junction at criticality (with the same $E_{\mathrm{J}} / E_{\mathrm{C}}$ and $\Delta \tau E_{\mathrm{C}}$ ). This suggests that many of the features of the NOR-FSC transition at the ICFP can be understood in terms of the single-junction Schmid transition, although the ICFP is an interacting fixed point. We will first present the numerical results and then proceed to discuss them in sections 5.2 and 6.

\subsection{Numerical results and resemblance to the single junction}

Associated with the drift of the phase boundary in the ICFP region as a function of $E_{\mathrm{J}} / E_{\mathrm{C}}$ (see figure 13) is a continuous change in the critical resistance of the junctions and in the value of the correlation exponents (defined below in equation (42)). As mentioned above, these features of the fixed point are remarkably similar in the two-junction model and in a single resistively shunted junction at criticality. To illustrate this, we first consider the critical resistance and plot in figure 16 the resistance from lead to central grain as a function of inverse temperature for $E_{\mathrm{J}} / E_{\mathrm{C}}=1, r=0.5 R_{\mathrm{Q}}$ and $R=0.65 R_{\mathrm{Q}}$, which is a point on the FSC-NOR phase boundary. Also shown in the figure is the temperature dependence of the resistance in a single junction (with $E_{\mathrm{J}} / E_{\mathrm{C}}=1$ and the 


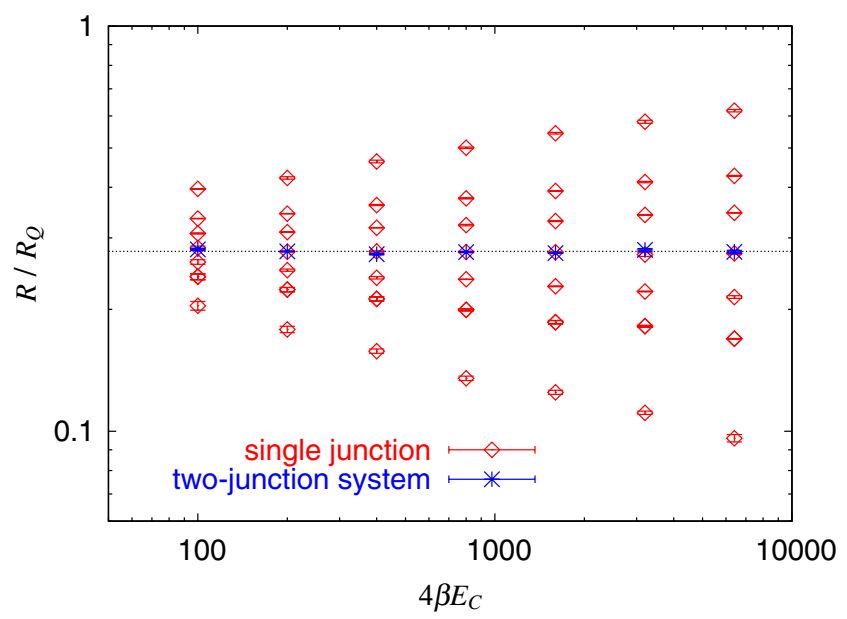

Figure 16. Critical lead-to-grain resistance as a function of inverse temperature for $E_{\mathrm{J}} / E_{\mathrm{C}}=1$ and $\Delta \tau E_{\mathrm{C}}=0.25$. The point $r=0.5 R_{\mathrm{Q}}, R=0.65 R_{\mathrm{Q}}$ on the FSC-NOR phase boundary has been selected (see figure 11). For comparison, we also plot resistance-versus-temperature data for a single resistively shunted junction with $E_{\mathrm{J}} / E_{\mathrm{C}}=1$ and $\Delta \tau E_{\mathrm{C}}=0.25$. The values $R_{\mathrm{S}}$ of the shunt resistors are (from top to bottom) $R_{\mathrm{Q}} / R_{\mathrm{S}}=0.9,0.95,0.975,1.0,1.025,1.05$, and 1.1, respectively. At the phase transition point $R_{\mathrm{Q}} / R_{\mathrm{S}}=1$, the resistance of the single junction is exactly the same as the critical lead-to-grain resistance in the two-junction system.

same discretization step $\left.\Delta \tau E_{\mathrm{C}}=0.25\right)$ for several values of the shunt resistance $R_{\mathrm{s}}$. The curves from top to bottom correspond to $R_{\mathrm{Q}} / R_{\mathrm{S}}=0.9,0.95,0.975,1.0,1.025,1.05$ and 1.1 , respectively. For $R_{\mathrm{Q}} / R_{\mathrm{S}}>1$, the junction turns superconducting as $T \rightarrow 0$, whereas for $R_{\mathrm{Q}} / R_{\mathrm{S}}<1$ it becomes insulating. At the critical point $R_{\mathrm{Q}} / R_{\mathrm{s}}=1$, the resistance of the system (junction plus shunt resistor) is precisely the same as the critical resistance from lead to central grain in the two-junction model.

By and large, the critical resistance of the two-junction system does not vary along the FSC-NOR phase boundary for fixed Josephson coupling. We plot its value in figure 17 as a function of $r$ and compare it with the corresponding result for a single resistively shunted junction. A very good agreement is evident for $r / R_{\mathrm{Q}}=0.1,0.2$, and 0.5. For $r / R_{\mathrm{Q}}=0.7$, closer to the meeting point of the three phases, the agreement is less good although still within error bars.

Another quantity we measured is the mean phase fluctuation $\left\langle(\phi-\bar{\phi})^{2}\right\rangle$, which we found in [14] to grow proportional to the logarithm of the inverse temperature at criticality. The same is true in the two-junction system. In figure 18 we plot $\left\langle(\phi-\bar{\phi})^{2}\right\rangle$ as a function of inverse temperature. The lines show the data for a single junction with the bold line marking the logarithmic growth of the phase fluctuations at the critical point. The diamonds, circles and triangles show the data obtained from the two-junction system at criticality. They correspond to points along the FSC-NOR phase boundary with $E_{\mathrm{J}} / E_{\mathrm{C}}=1$ and $\Delta \tau E_{\mathrm{C}}=0.25$, as in the single-junction case. The uncertainty on the critical value of $R$ has not been taken into account in the error bars of figure 18. This uncertainty can account for the small deviations between the different data sets. 
Simulation results for an interacting pair of resistively shunted Josephson junctions

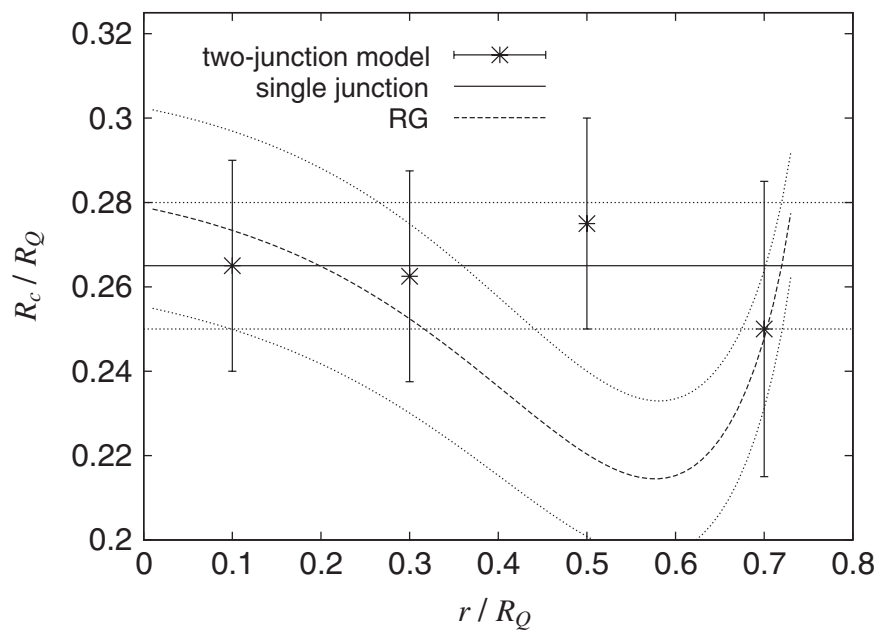

Figure 17. Value of the critical lead-to-grain resistance along the FSC-NOR phase boundary for $E_{\mathrm{J}} / E_{\mathrm{C}}=1$. The critical resistance is nearly independent of $r$ and within error-bars it is the same as in a single junction. The solid line shows the critical resistance of the single resistively shunted junction with $E_{\mathrm{J}} / E_{\mathrm{C}}=1$ and $R_{\mathrm{S}}=R_{\mathrm{Q}}$. Error estimates are indicated by the dotted lines. The dashed line is the result of an RG-based calculation of the effective lead-to-grain resistance.

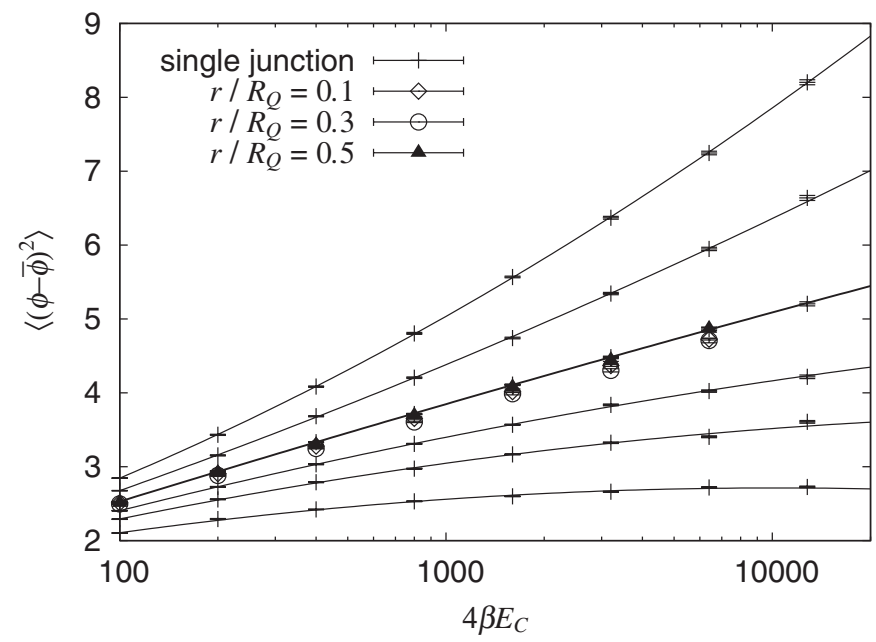

Figure 18. Phase fluctuations $\left\langle(\phi-\bar{\phi})^{2}\right\rangle$ as a function of inverse temperature. The curves from top to bottom show the data for a single resistively shunted junction with $R_{\mathrm{Q}} / R_{\mathrm{S}}=0.9,0.95,1.0,1.05,1.1$, and 1.2 respectively. The symbols show the same quantity calculated in the two-junction model at criticality. Three points along the FSC-NOR phase boundary have been chosen and all the data agree with those of the single junction with identical values of $E_{\mathrm{J}} / E_{\mathrm{C}}=1$ and $\Delta \tau E_{\mathrm{C}}=0.25$.

In addition, we considered the exponents $\eta(q)$, which we measured for several values of $E_{\mathrm{J}} / E_{\mathrm{C}}$ at $r=0.5 R_{\mathrm{Q}}$ and with $R / R_{\mathrm{Q}}$ corresponding to the FSC-NOR phase boundary. 


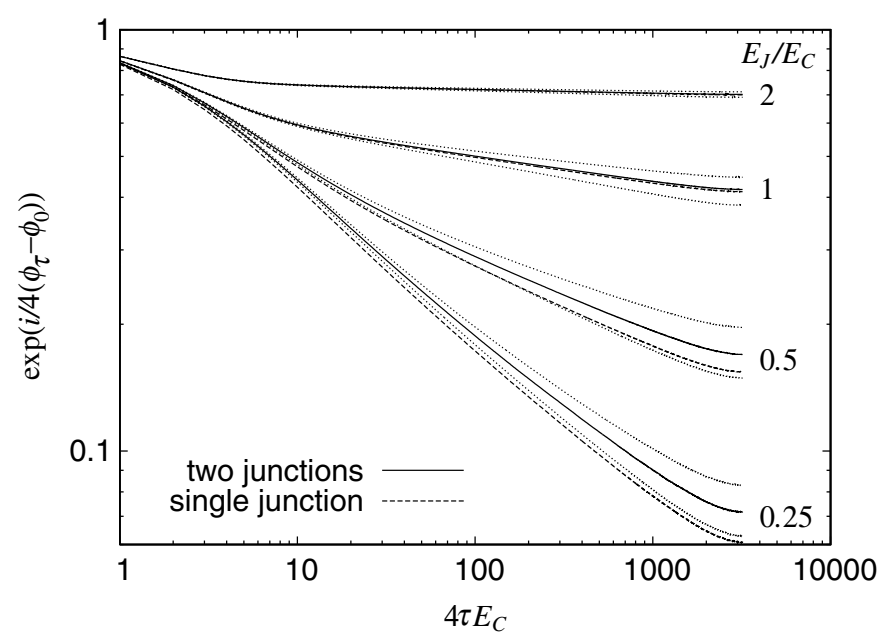

Figure 19. Correlation function (42) for $q=0.25$ and $\beta E_{\mathrm{C}}=1600$. The curves correspond to $r=0.5 R_{\mathrm{Q}}$, and from top to bottom $E_{\mathrm{J}} / E_{\mathrm{C}}=2,1,0.5$ and 0.25 , respectively; $R$ in these plots is tuned to the FSC-NOR transition point. The solid lines show the correlation functions computed for the two-junction system and the dashed lines those obtained for a single resistively shunted Josephson junction at criticality. The dotted lines indicate the error of the two-junction calculation originating from the uncertainty on the critical value of $R$.

We define the latter as in [13] using the correlation function

$$
\operatorname{corr}_{q}(\tau) \equiv\langle\exp [\mathrm{i} q(\phi(\tau)-\phi(0))]\rangle,
$$

with $q$ some non-integral real number and $\phi$ the phase difference across one of the junctions. In the normal phase and at criticality the correlations decay as

$$
\operatorname{corr}_{q}(\tau) \sim \tau^{-2 \eta(q)} .
$$

In the superconducting phase one observes a power-law decay of the connected correlation function

$$
\operatorname{corr}_{q}(\tau) \equiv\langle\exp [\operatorname{iq}(\phi(\tau)-\phi(0))]\rangle-|\langle\exp [\operatorname{iq} \phi(\tau)]\rangle|^{2} .
$$

In figure 19 we plot $\operatorname{corr}_{q=1 / 4}(\tau)$ for the Josephson coupling strengths $E_{\mathrm{J}} / E_{\mathrm{C}}=0.25,0.5,1$, 2 (as in figure 13) and the corresponding critical resistances $r=0.5$ and $R=0.5375(200)$, $0.5875(150), 0.650(20), 0.6875(200)$, respectively. We compare these correlation functions to those obtained for a single junction at criticality $\left(R_{\text {shunt }}=R_{\mathrm{Q}}\right)$ for the same values of $E_{\mathrm{J}} / E_{\mathrm{C}}$ and the same discretization step $\Delta \tau E_{\mathrm{C}}=0.25$. As can be seen in figure 19, the correlation functions, and thus also the critical exponents $\eta(q)$, perfectly agree for $E_{\mathrm{J}} / E_{\mathrm{C}}=2$ and 1 , while they agree within error-bars for $E_{\mathrm{J}} / E_{\mathrm{C}}=0.5$ (the dotted lines in the figure show the correlation functions computed at $R=R_{\text {critical }} \pm$ error). For $E_{\mathrm{J}} / E_{\mathrm{C}}=0.25$ the agreement is no longer as good, but the exponents for a single junction and for two junctions remain close and well within error-bars.

While the exponent $\eta(q)$ varies as a function of $E_{\mathrm{J}} / E_{\mathrm{C}}$ in a remarkably similar way as in a single junction, there is no dependence of this exponent for fixed $E_{\mathrm{J}} / E_{\mathrm{C}}$ on the value of $r$ and $R$, that is on the position along the FSC-NOR phase boundary shown in 
Simulation results for an interacting pair of resistively shunted Josephson junctions

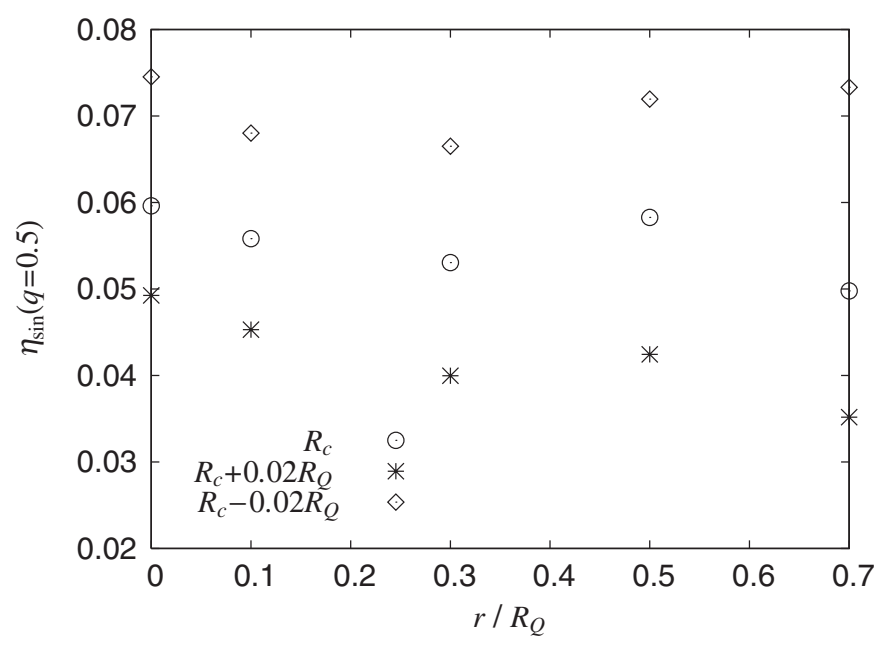

Figure 20. Correlation exponents $\eta_{\sin }(q=0.5)$ obtained from a fit to $f(\tau)=$ $a / \sin (\pi / \beta \tau)^{2 \eta_{\sin }}$ along the FSC-NOR phase boundary (see figure 11) for constant $E_{\mathrm{J}} / E_{\mathrm{C}}=1$ and $\beta E_{\mathrm{C}}=1600$. The main source of error is the uncertainty of $\pm 0.02 R_{\mathrm{Q}}$ on the critical resistance $R_{\mathrm{c}}$ for a given value of $r$. The independence of the exponent $\eta_{\sin }(q=0.5)$ with respect to $r$ and the constant lead-to-grain resistance shown in figure 17 indicate that the critical properties only depend on the value of $E_{\mathrm{J}} / E_{\mathrm{C}}$.

figure 11. Remember that also the effective lead-to-grain resistance was within error-bars independent of $r$ on the FSC-NOR phase boundary (figure 17).

We plot $\eta_{\sin }(q)$, obtained from a fit to

$$
f(\tau)=\frac{a}{\sin ((\pi / \beta) \tau)^{2 \eta_{\sin }}}
$$

along the FSC-NOR phase boundary for $q=0.5, \beta E_{\mathrm{C}}=1600$ and $E_{\mathrm{J}} / E_{\mathrm{C}}=1$ in figure 20 . The measured exponents range between 0.05 and 0.06 and are thus well within error-bars. To estimate the error, which mainly originates from the uncertainty of $\pm 0.02 R_{\mathrm{Q}}$ on the critical value of $R_{\mathrm{c}}$ (for a given $r$ ), we also plot the exponents measured at $R_{\mathrm{c}} \pm 0.02 R_{\mathrm{Q}}$ in the figure.

\subsection{RG analysis of the critical effective resistance in the two-junction system}

The extensive investigation in the previous section demonstrated a remarkable resemblance in the critical behaviour between the two-junction system and a single junction with the same $E_{\mathrm{J}} / E_{\mathrm{C}}$. In this section we will account for this resemblance by comparing the measured effective resistance at criticality of the two-junction system with the resistance as predicted from the RG flow equations (15) and (16). We emphasize that such an analysis is only approximate, since equations (15) and (16) are derived in the limit of large $E_{\mathrm{J}} / E_{\mathrm{C}}$, and the case we are concentrating on is that of an intermediate $E_{\mathrm{J}} / E_{\mathrm{C}}$. Nevertheless, the strong coupling RG equations provide a rather good description of the behaviour of the system at criticality. Since all quantities considered in section 5.1 (lead-to-grain resistance, phase fluctuations, and phase correlations) are related to the effective lead-to-grain resistance, the analysis of the latter suffices. 


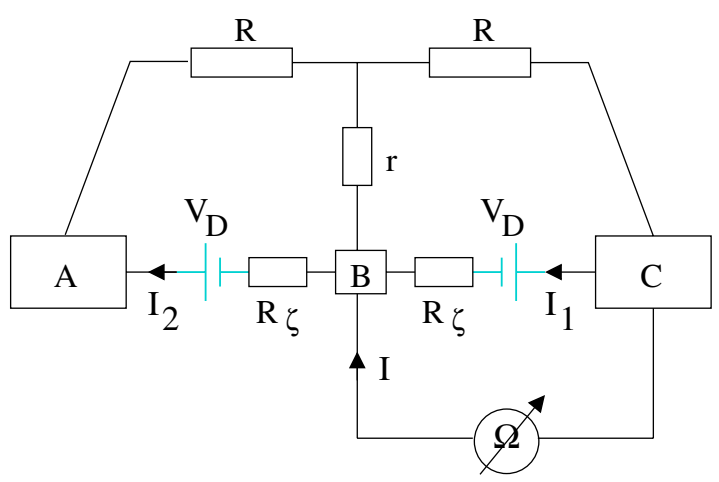

Figure 21. A circuit illustrating the measurement of the lead-to-grain resistance. Each junction is replaced by two components: a resistor $R_{\zeta}=\alpha \zeta^{2}$ which describes the response of single phase-slips, and a voltage drop $V_{\mathrm{D}}$ which in junction $\mathrm{BC}$ is $\alpha \zeta_{\mathrm{D}}^{2}\left(I_{1}-I_{2}\right)$ and in junction $\mathrm{AB}$ is $\alpha \zeta_{\mathrm{D}}^{2}\left(I_{2}-I_{1}\right)$. The ohmmeter is considered as a current source, $I$.

The first step in the analysis is to connect the measured lead-to-grain effective resistance with the resistances $R$ and $r$, and with the phase-slip fugacities $\zeta$ and $\zeta_{\mathrm{D}}$ (which we will later substitute with their fixed-point values, $\zeta^{*}$ and $\zeta_{\mathrm{D}}^{*}$ ). This amounts to solving the circuit shown in figure 21. The junctions in the figure were each replaced by two components: a resistor

$$
R_{\zeta} \approx \alpha \zeta^{2}
$$

and a voltage drop due to phase-slip dipoles, $V_{\mathrm{D}}$. The latter is given by

$$
V_{\mathrm{D}} \approx \alpha \zeta_{\mathrm{D}}^{2}\left(I_{1}-I_{2}\right)
$$

The solution of the circuit in figure 21 is sketched in appendix C. It leads to the following expression for the lead-to-grain resistance:

$$
R_{\mathrm{eff}}=\frac{\left(R^{2}+2 r R\right)\left(\zeta^{2}+\zeta_{\mathrm{D}}^{2}\right)+(R+r) \alpha \zeta^{2}\left(\zeta^{2}+2 \zeta_{\mathrm{D}}^{2}\right)}{\left(R / \alpha+\zeta^{2}\right)\left(2 r+R+\alpha \zeta^{2}+2 \alpha \zeta_{\mathrm{D}}^{2}\right)} .
$$

In equations (46) and (47) we used the coefficient $\alpha$ to convert the square of the phaseslip fugacities to resistances. This coefficient is going to be the only input into the RG flow analysis of the effective lead-to-grain resistance. An estimate for this coefficient can be obtained from the data point at $E_{\mathrm{J}} / E_{\mathrm{C}}=1$ and $r / R_{\mathrm{Q}}=0.5$ in figure 17 , which coincides with the measured single-junction effective resistance. The critical shunt resistance at this point is $R / R_{\mathrm{Q}}=0.65 \pm 0.02$. From the integrated $\mathrm{RG}$ flow we can determine $\zeta_{0}$ such that for $r=0.5 R_{\mathrm{Q}}$ and $R=0.65 R_{\mathrm{Q}}$ the point $\left(\zeta_{0}, 0\right)$ lies on the critical manifold. We find

$$
\zeta_{0}=0.295 \pm 0.01 \text {. }
$$

This $\zeta_{0}$ corresponds to $E_{\mathrm{J}} / E_{\mathrm{C}}=1$. Note that $\zeta_{0}$ is a function of $E_{\mathrm{J}} / E_{\mathrm{C}}$ only in the strong coupling limit, and should only depend weakly on the resistances $r, R$ (cf [16]). The same applies to the coefficient $\alpha$. Bearing this in mind, we can calculate $\alpha$ by using the measured resistance of the single-junction system. This measured resistance is the resistance of the 
junction, $\alpha \zeta^{2}$, parallel to the shunt resistor, which equals $R_{\mathrm{Q}}$ at criticality. Thus, for $E_{\mathrm{J}} / E_{\mathrm{C}}=1$ we have (figure 17 )

$$
\frac{\alpha \zeta_{0}^{2} \cdot R_{\mathrm{Q}}}{R_{\mathrm{Q}}+\alpha \zeta_{0}^{2}}=0.265 \pm 0.015
$$

from which we find

$$
\alpha=4.15 \pm 0.35 .
$$

Now we have all the pieces to predict the effective lead-to-grain resistance, $R_{\text {eff }}$, on the FSC-NOR critical line $E_{\mathrm{J}} / E_{\mathrm{C}}=1$. By using equation (48) with the fixed-point values $\zeta^{*}$ and $\zeta_{\mathrm{D}}^{*}$ from equation (27), and $\alpha$ from equation (51), we obtain the curve of $R_{\text {eff }}$ as a function of $r$. The result of this calculation is shown in figure 17 by the dashed line. One can see that the resistance predicted by the RG changes little in the entire range, and remains in reasonably good agreement with the observed Monte Carlo $R_{\text {eff }}$.

Note that we assumed that the measured resistance is due to the fixed-point characteristics of the two-junction system. From section 4.2, however, we know that in the vicinity of $r=0.75 R_{\mathrm{Q}}$, where the three phases meet, crossover effects are dominant. Therefore we expect the lead-to-grain resistance calculated in this section to deviate from the measured $R_{\text {eff }}$ in that vicinity. Another caveat for the current calculation is that it is correct up to second order in the phase-slip fugacities; fourth-order contributions to $R_{\text {eff }}$ are neglected (although we keep fourth-order terms in equation (48)). These corrections may also account for deviations from the measured $R_{\text {eff }}$.

\section{The ICFP as a self-consistent fixed point}

In the previous section we investigated the FSC-NOR transition extensively, and demonstrated a remarkable resemblance between the two-junction and single-junction systems at criticality. We adequately explained this surprising resemblance using the $\mathrm{RG}$ from section 2. In this section, however, we provide yet another explanation, albeit ad hoc, for this resemblance. The alternative explanation is that the ICFP can be approximated as a self-consistent fixed point. In such a mean-field theory, illustrated in figure 22 , the physics of a single junction emerges naturally. The idea behind this approach is that a Josephson junction undergoes an SC-NOR transition when its effective shunting resistance is $R_{\mathrm{Q}}$.

Consider the two-junction system with phase-slip fugacities $\zeta_{0}\left(\right.$ with $\zeta_{\mathrm{D}}=0$ ) in both junctions. In mean field, one junction sees the other as an effective resistor (figures 22 and 24) with resistance

$$
R_{\mathrm{J}}=\alpha \zeta_{0}^{2},
$$

where $R_{\mathrm{J}}$ is defined from equation (50). Therefore the effective shunting resistance on each of the junctions is

$$
R_{\mathrm{s}} \approx R+\frac{r\left(R+R_{\mathrm{J}}\right)}{r+R+R_{\mathrm{J}}}
$$

and criticality is obtained when

$$
R_{\mathrm{s}}=R_{\mathrm{Q}} .
$$



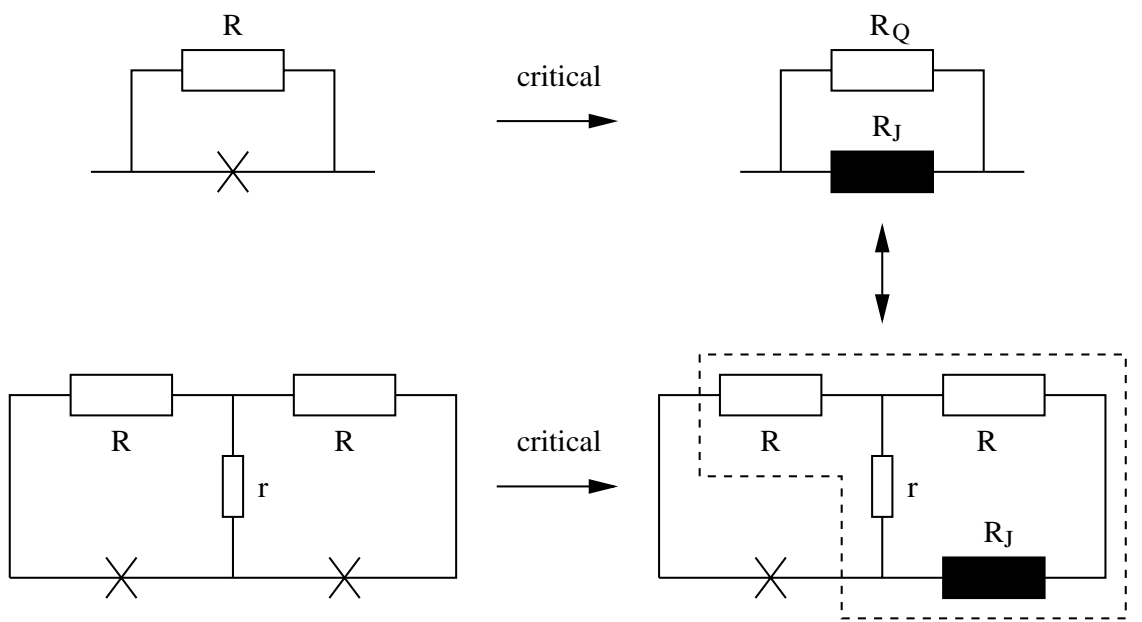

Figure 22. Illustration of the mean-field theory for the FSC-NOR transition in the two-junction model. A Josephson junction at criticality is replaced by a resistor $R_{\mathrm{J}}$, such that the resistance of the circuit on the top right corresponds to the measured resistance of the device. Replacing one of the junctions in the two-junction system by $R_{\mathrm{J}}$, one can calculate the effective shunt resistance $R_{\mathrm{s}}^{\mathrm{eff}}$ of the other junction. Identifying $R_{\mathrm{s}}^{\text {eff }}$ with $R_{\mathrm{Q}}$ yields an equation for the FSC-NOR phase boundary.

From equations (53) and (54) we find an expression for the FSC-NOR phase boundary,

$$
r=\frac{\left(R+R_{\mathrm{J}}\right)\left(R_{\mathrm{Q}}-R\right)}{2 R+R_{\mathrm{J}}-R_{\mathrm{Q}}}
$$

as a function of the parameter $R_{\mathrm{J}}$, which depends on $E_{\mathrm{J}} / E_{\mathrm{C}}$. The limiting cases for strong and weak Josephson potential work out correctly. For $E_{\mathrm{J}} / E_{\mathrm{C}} \rightarrow \infty, R_{\mathrm{J}} \rightarrow 0$ (superconducting junction) and equation (55) reduces to

$$
r=\frac{R\left(R_{\mathrm{Q}}-R\right)}{2 R-R_{\mathrm{Q}}}
$$

whereas for $E_{\mathrm{J}} / E_{\mathrm{C}} \rightarrow 0, R_{\mathrm{J}} \rightarrow \infty$ (insulating junction) and we obtain

$$
r=R_{\mathrm{Q}}-R \text {. }
$$

These are indeed the equations describing the FSC-NOR phase boundaries shown in figure 2.

In the mean-field approximation, each junction at criticality behaves as an independent junction, and thus exhibits the same phase correlations and fluctuations as the single junction with the same $R_{\mathrm{J}}$, and hence exhibits the same dependence on $E_{\mathrm{J}} / E_{\mathrm{C}}$.

Although this explanation seems naive, it does allow a simple and quantitative understanding of the observed (and calculated) properties of the two junction-system at the FSC-NOR transition point. As further evidence, in figure 23, we compare the phase boundaries in the ICFP region as obtained by the simple mean-field treatment (equation (55)), the RG calculation (explained in appendix B), and the Monte Carlo results. For $r / R_{\mathrm{Q}}<0.7$ there is a good agreement between the three approaches. In the 
Simulation results for an interacting pair of resistively shunted Josephson junctions
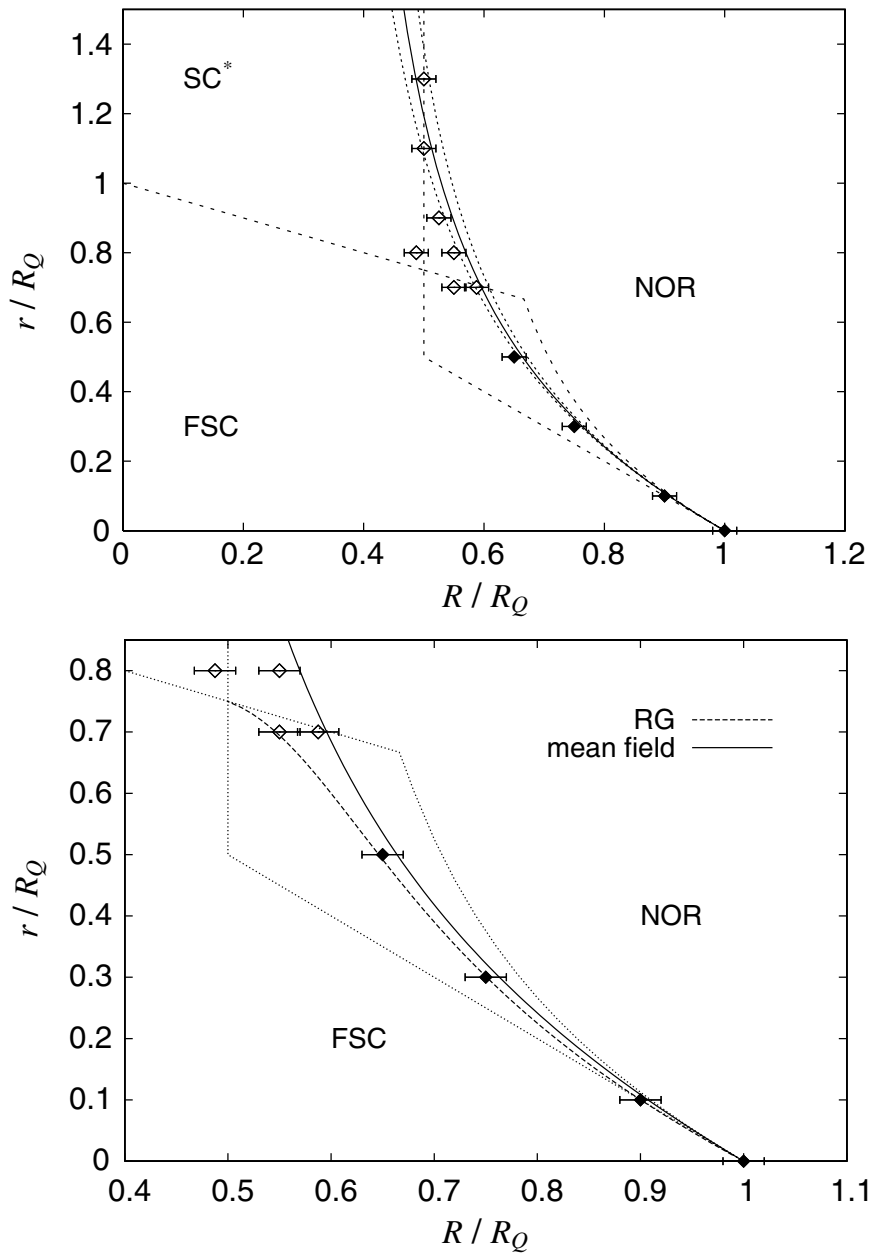

Figure 23. Upper figure: FSC-NOR phase boundary obtained from the meanfield prediction equation (55) for $E_{\mathrm{J}} / E_{\mathrm{C}}=1$. The critical resistance $R_{\mathrm{c}}$ of the single junction with $R_{\mathrm{S}}=R_{\mathrm{Q}}$ is $R_{\mathrm{c}} / R_{\mathrm{Q}} \approx 0.265$ (see figure 17). The dotted lines show how the error on $R_{\mathrm{c}}$ influences the outcome. A remarkably good agreement with the Monte Carlo results is obtained. Lower figure: a close-up of the phase diagram in the ICFP region for $E_{\mathrm{J}} / E_{\mathrm{C}}=1\left(\zeta_{0}=0.295\right)$. The solid line is the mean-field phase boundary from equation (55) and the dashed line is the RG prediction for the FSC-NOR phase boundary. The RG result agrees well with the mean-field prediction and with the Monte Carlo results for $r<0.6 R_{\mathrm{Q}}$. Filled dots show the position of the FSC-NOR transition, and open dots indicate an FSC-SC* or SC ${ }^{*}-\mathrm{NOR}$ transition, respectively. See section 4.2 for a discussion of crossover effects.

vicinity of the meeting point of the three phases, the RG boundary traces the measured FSC-SC* phase boundary, while the mean-field prediction agrees with the Monte Carlo $\mathrm{SC}^{*}-\mathrm{NOR}$ phase boundary, even for values of $r>0.75 R_{\mathrm{Q}}$. Note, though, that despite the agreement with the Monte Carlo results, the mean-field argument does not apply to the $\mathrm{SC}^{*}-\mathrm{NOR}$ phase boundary.

The good agreement of the mean-field boundary with the measured FSC-NOR boundary seems rather surprising. It can be explained once we understand why the 


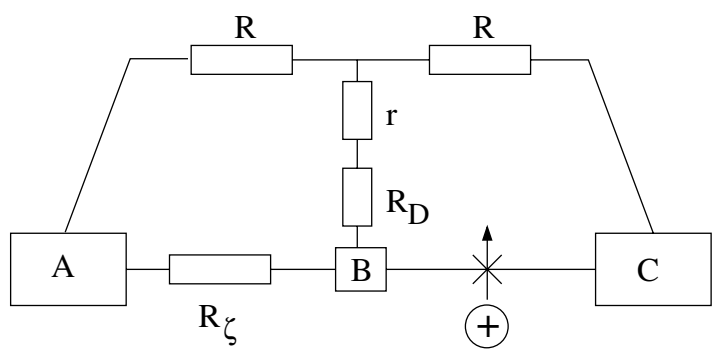

Figure 24. Effective circuit in the mean-field approximation-taking phase-slip dipoles into account. Junction AB is approximated by a resistor with resistance $R_{\zeta}=\alpha \zeta^{2}$ which is due to single phase slips. In addition, phase-slip dipoles add $R_{\mathrm{D}}=\alpha \zeta_{\mathrm{D}}^{2}$ to the resistor $r$. The location of $R_{\mathrm{D}}$ is determined such that it responds to the difference in the current on the two junctions (cf equation (47)). This circuit is equivalent to the one shown in figure 21 with $I=0$. In the meanfield approximation we assume that a NOR-FSC phase transition occurs when the effective shunting resistance that a phase slip on the junction $\mathrm{BC}$ probes equals $R_{\mathrm{Q}}$. Furthermore, we assume that $R_{\mathrm{D}}$ is negligible - an assumption which is justified by the Monte Carlo results in the ICFP region and near the meeting point of the three phases, where crossover effects are evident.

mean-field treatment is (at least qualitatively) reasonable. The mean-field approach relies on two assumptions: (1) a junction with phase-slip fugacity $\zeta$ can be replaced by a resistor $\alpha \zeta^{2}$, and (2) $\alpha \zeta_{\mathrm{D}}^{2}$ can be neglected compared to $r$ (see figure 24). At this point we can only justify the first assumption on qualitative, but not on analytical, grounds. Assumption (2) on the other hand is justified by the fact that the fixed-point values of the phase-slip fugacities obey $\alpha\left(\zeta_{\mathrm{D}}^{*}\right)^{2} \ll r$ for much of the ICFP region. For $\zeta_{0}=0.295$ (corresponding to $\left.E_{\mathrm{J}} / E_{\mathrm{C}}=1\right)$ this ratio rises slowly from zero at $r=0$ to 0.25 at $r=0.6 R_{\mathrm{Q}}$; it crosses 1 at $r=0.73 R_{\mathrm{Q}}$. In the region where $\alpha\left(\zeta_{\mathrm{D}}^{*}\right)^{2}$ is no longer negligible, i.e., $r>0.6 R_{\mathrm{Q}}$, crossover effects set in, and the RG flow is cut off at the lowest energy scale of the Monte Carlo simulation. In this case $\zeta_{\mathrm{D}}$ does not reach its fixed-point value and will be observed as small. The crossover effects justify assumption (2) of the mean-field calculation in the same way that they explain the deviations from the predicted phase diagram in section 4.2. Thus the mean-field approach reproduces the phase diagram with crossover effects.

Above we made the assumption that in the mean-field approach a junction undergoes the $\mathrm{SC}-\mathrm{NOR}$ transition when the effective shunting resistance equals $R_{\mathrm{Q}}$. We also derived the phase boundary using the RG flow (appendix B) without making any such assumption. This raises the question: how close to $R_{\mathrm{Q}}$ is the effective shunting resistance along the RG-predicted phase boundary? This effective shunting resistance is given by

$$
R_{\mathrm{s}}^{\mathrm{eff}} \approx R+\frac{\left(R+R_{\zeta}\right)\left(r+R_{\mathrm{D}}\right)}{r+R+R_{\zeta}+R_{\mathrm{D}}}
$$

which can be deduced by observation from figure 24, with $R_{\mathrm{D}}=\alpha \zeta_{\mathrm{D}}^{2}$ and $R_{\zeta}=\alpha \zeta^{2}$. In figure 25 we plot this effective shunting resistance for $\zeta_{0}=0.295$, which corresponds to $E_{\mathrm{J}} / E_{\mathrm{C}}=1$, as a function of $r$ for the entire ICFP region, $0<r / R_{\mathrm{Q}}<0.75$. We assume that $\zeta$ and $\zeta_{\mathrm{D}}$ take the fixed-point values defined in equations (27). As can be 


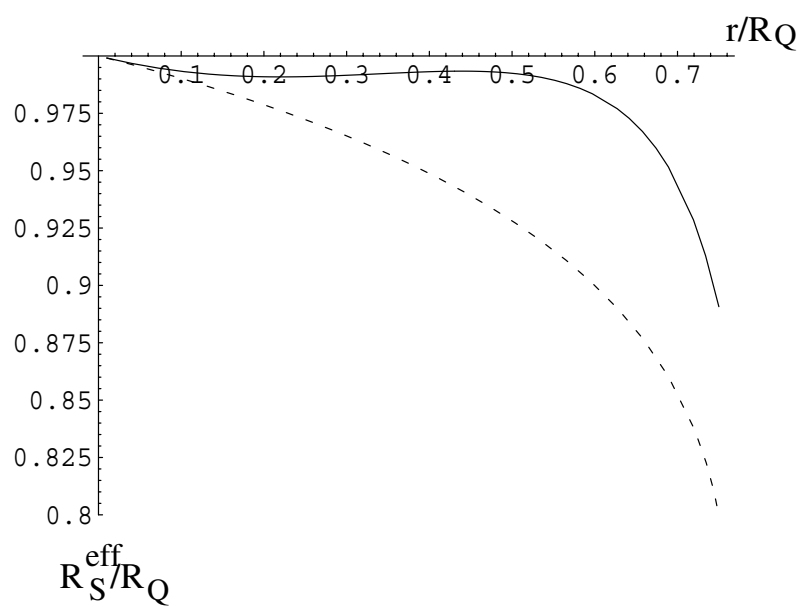

Figure 25. The RG-based calculation of $R_{\mathrm{S}}^{\text {eff }}$ (solid line) given in equation (58) as a function of $r / R_{\mathrm{Q}}$ on the phase boundary in the ICFP region with $E_{\mathrm{J}} / E_{\mathrm{C}}=1$ $\left(\zeta_{0}=0.295\right)$. The effective shunt resistance is very close to $R_{\mathrm{Q}}$ in the entire region, giving more validity to the proposed mean-field approach to the double sine-Gordon model of the two-junction system. The dotted line is the effective shunt resistance when we set $R_{\zeta}=0$ and $R_{\mathrm{D}}=0$, and is given for reference (this is just $R+r R /(r+R))$.

seen, the effective shunting resistance stays very close to $R_{\mathrm{Q}}$ over the entire range. At this point we are unable to say whether this is just a coincidence (as the success of the meanfield treatment above may therefore be), or a universal property of the phase boundary, which the approximate $\mathrm{RG}$ roughly reproduces. To answer this question, a more thorough consideration of the sine-Gordon model is necessary.

\section{Correlation function in the NOR phase}

In this section we compute and briefly consider the decay of the correlation function (see equation (42))

$$
\operatorname{corr}_{q}(\tau) \equiv\langle\exp [\mathrm{i} q(\phi(\tau)-\phi(0))]\rangle
$$

in the NOR phase of the system. We set $r=0.5 R_{\mathrm{Q}}$ and $R=0.65 R_{\mathrm{Q}}$, and calculate $\operatorname{corr}_{q}(\tau)$ for different values of the Josephson coupling energy. This choice of $r$ and $R$ corresponds to a critical value $\left(E_{\mathrm{J}} / E_{\mathrm{C}}\right)_{\text {critical }}=1$ (see figure 13 ). Our results are shown in figure 26. Interestingly, each curve has an approximate power-law behaviour, but with exponents that vary with $E_{\mathrm{J}} / E_{\mathrm{C}}$. The correlation exponents $\eta_{\sin }(q=0.5)$ obtained from a fit to equation (45) are shown in figure 27 for the temperatures $\beta E_{\mathrm{C}}=1600,800$ and 400 . This behaviour is very different from that seen in a single resistively shunted junction, where different $E_{\mathrm{J}} / E_{\mathrm{C}}$ (at fixed shunt resistance $R_{\mathrm{s}}$ ) yield the same exponents $\eta$ in the NOR phase [14].

However, one point should be kept in mind: even though in the previous section we demonstrated and discussed the similarities between the two-junction model and the single junction at criticality, there are also stark differences between the two systems. While in the single junction, flow lines corresponding to systems with the same shunt resistance but 


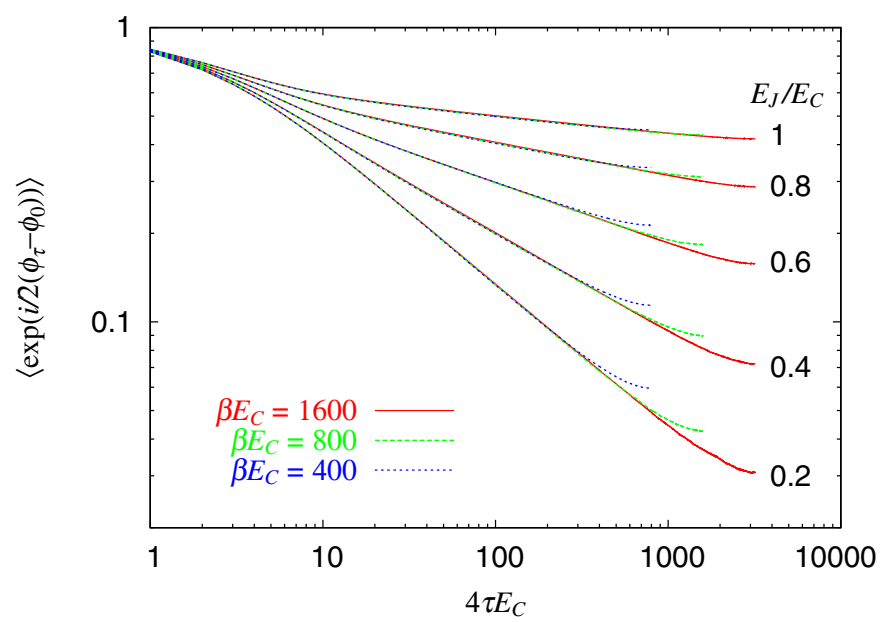

Figure 26. Correlation functions $\operatorname{corr}_{q=0.5}(\tau)$ in the FSC phase for different temperatures and values of $E_{\mathrm{J}} / E_{\mathrm{C}}$, but fixed resistance $r=0.5 R_{\mathrm{Q}}$ and $R=0.65 R_{\mathrm{Q}}$ (corresponding to $\left.\left(E_{\mathrm{J}} / E_{\mathrm{C}}\right)_{\text {critical }}=1\right)$. Different $E_{\mathrm{J}} / E_{\mathrm{C}}$ yield considerably different exponents $\eta(q)$.

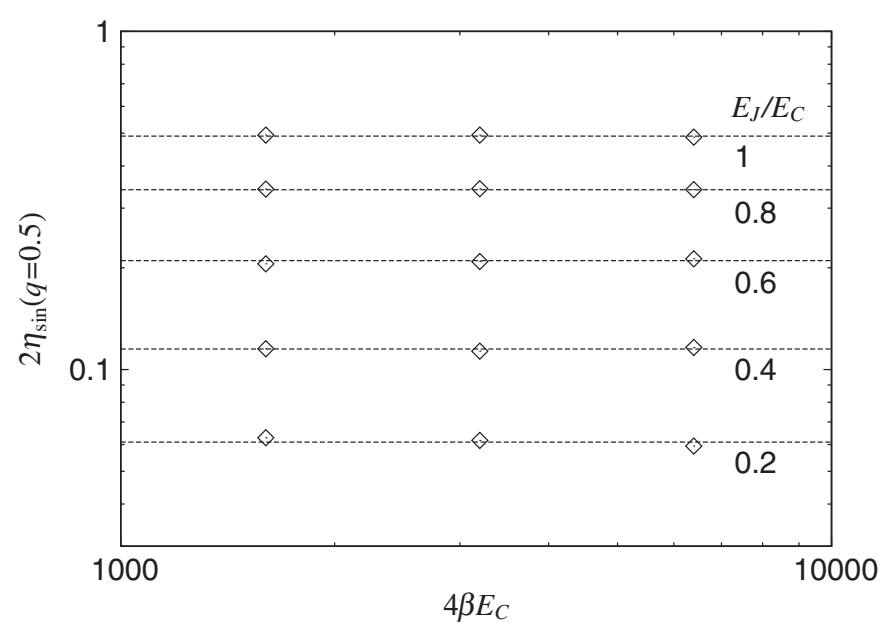

Figure 27. Correlation exponents $\eta_{\sin }(q=0.5)$ obtained from a fit to equation (45) for the temperatures $\beta E_{\mathrm{C}}=1600,800$ and 400.

different $E_{\mathrm{J}} / E_{\mathrm{C}}$ merge (as they flow parallel to the $E_{\mathrm{J}} / E_{\mathrm{C}}$ axis only), in the two-junction system, under the same circumstances, they do not overlap (see figure 7 ). Indeed, in equations (8), (9) and (15), (16) we need an extra parameter, $J_{+}$and $\zeta_{\mathrm{D}}$ respectively, in order to write the renormalization group flow equations for the two-junction system.

In the single-junction model we expect systems with different $J \propto E_{\mathrm{J}} / E_{\mathrm{C}}$ but the same shunt resistance to show the same low-energy behaviour. More specifically, at weak $J$ we expect

$$
\operatorname{corr}_{q}(\tau, J)=b^{-2 q^{2}\left(R / R_{\mathrm{Q}}\right)} \operatorname{corr}_{q}\left(\frac{\tau}{b}, \frac{J}{b^{R / R_{\mathrm{Q}}-1}}\right) .
$$




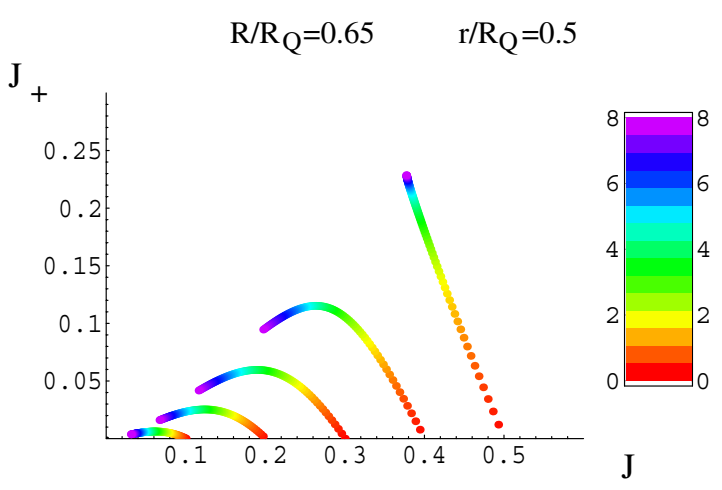

Figure 28. The integrated weak coupling RG flows of equations (20) and (21) in the $\left(J, J_{+}\right)$plane for $R=0.65 R_{\mathrm{Q}}$ and $r=0.5 R_{\mathrm{Q}}$. The value $J_{0}=0.5$ corresponds to the critical value $E_{\mathrm{J}} / E_{\mathrm{C}}=1$. These flows show qualitatively how the paths with different bare coupling $J_{0}$ do not overlap, and also do not reach zero in the accessible energy range. The flows are colour coded to show the evolution of the logarithmic RG scale $l=\ln \Lambda_{0} / \Lambda$ along the flow $\left(\Lambda_{0}\right.$ is the bare UV cutoff and $\Lambda$ is the renormalized cutoff). In figure $26 l \leq 8$, as in this plot.

To compare two different systems, with junctions $J_{1}$ and $J_{2}$, we can choose

$$
b=\left(\frac{J_{1}}{J_{2}}\right)^{1 /\left(R / R_{\mathrm{Q}}-1\right)}
$$

and obtain

$$
\operatorname{corr}_{q}\left(\tau, J_{1}\right)=\left(\frac{J_{1}}{J_{2}}\right)^{-2 q^{2} R /\left(R-R_{\mathrm{Q}}\right)} \operatorname{corr}_{q}\left(\tau\left(\frac{J_{1}}{J_{2}}\right)^{1 /\left(R / R_{\mathrm{Q}}-1\right)}, J_{2}\right) .
$$

If the decay of the correlations is a power law, $\operatorname{corr}_{q}(\tau) \sim|\tau|^{-2 \eta(q)}$, then the scaling relation (61) clearly shows that $J$ must drop out of the power law. In fact, from equation (59) we find directly

$$
\eta(q)=q^{2} \frac{R}{R_{\mathrm{Q}}}
$$

In the two-junction system, we observe a power-law decay of $\operatorname{corr}_{q}\left(\tau, J_{1}\right)$, but with $\eta(q)$ which seemingly depends also on $J$. In the absence of our understanding of the RG flows of the two-junction system, we might guess that a scaling relation similar to equation (59) holds, but with the shunt resistors $R$ and $r$ also flowing. This possibility, however, seems to contradict [3], which claims that in sine-Gordon models the resistance can not get renormalized due to its singular $|\omega|$-frequency dependence.

We can, however, qualitatively explain the dependence of $\eta(q)$ on $J$ in terms of the RG flow equations (20) and (21). In terms of the two-junction RG flows for $J$ and $J_{+}$ (or $\zeta$ and $\zeta_{\mathrm{D}}$ ), a scaling form such as equation (61) does not hold in the two-junction system in the ICFP region. Instead, we need to integrate equations (20) and (21). In figure 28 , the $\mathrm{RG}$ flows corresponding to $R=0.65 R_{\mathrm{Q}}, r=0.5 R_{\mathrm{Q}}$, and $J_{0}=0.5,0.4,0.3,0.2$ $\left(E_{\mathrm{J}} / E_{\mathrm{C}}=1,0.8,0.6,0.4\right)$, are shown. In the accessible energy range, all flows take different 
paths in the $\left(J, J_{+}\right)$space. Also, they are all far from saturating at $J=J_{+}=0$. The latter observation provides the reason for the slower decay of the correlations for higher $J$ : in the accessible energy range the two-junction system seems to have a larger effective $J$ and $J_{+}$, the higher the bare $J_{0}$ is. These finite $J$ and $J_{+}$, in turn, provide extra conductance in the system, and thus strengthen phase coherence, and suppress the decay of correlations.

We furthermore note that the flow in the temperature range $400<\beta E_{\mathrm{C}}<1600$ (corresponding to $6<l<7.4$ ) is rather slow, which might explain why there is hardly any temperature dependence in the measured $\eta$ (figure 27). However, we cannot rule out a scenario where the flows have actually converged to a set of distinct fixed points (with for example different values of $J_{+}$), although the origin of such additional fixed points remains unclear.

\section{Conclusions}

In this paper, we have presented an RG analysis and simulation results for a symmetric two-junction system. The phase diagram was found to contain all three phases predicted by theory: (i) fully superconducting (FSC), (ii) normal (NOR) and (iii) superconducting from lead to lead but normal from lead to grain $\left(\mathrm{SC}^{*}\right)$. Furthermore, the phase boundaries obtained by the Monte Carlo simulation agreed with the theoretical prediction, except for deviations near the tricritical point where the three phases meet. These deviations were interpreted as finite-temperature crossover effects, and give indirect evidence for the existence of the intermediate coupling fixed point. The behaviour of correlation functions in the NOR phase may also be indirect evidence for the flow of the coupling $J_{+}$, as predicted by the RG theory.

The two-junction system possesses the unusual ICFP region, in which the NOR-FSC transition can be tuned by $E_{\mathrm{J}} / E_{\mathrm{C}}$ as well as by the dissipation strength. We calculated this phase boundary using the RG treatment of [1], and found that it fits the Monte Carlo result quite well (cf figure 12). Theoretically it was shown that in this region the properties of the two-junction system are determined by a new intermediate coupling fixed point, with continuously varying exponents.

An efficient Monte Carlo algorithm was used to thoroughly investigate the intriguing ICFP region and the NOR-FSC phase transition. We calculated the lead-to-grain resistance, average phase fluctuations, and phase correlations for several locations on the critical NOR-FSC manifold. This manifold is two dimensional, and can be parameterized by $r$ and $E_{\mathrm{J}} / E_{\mathrm{C}}$. For the various sets of $\left(r, E_{\mathrm{J}} / E_{\mathrm{C}}\right)$ on the NOR-FSC critical manifold we made a surprising observation: the effective fixed-point lead-to-grain resistance, $R_{\mathrm{AB}}$, as well as other critical properties of the two-junction model, were nearly identical to those of a single junction (with the same $\left.E_{\mathrm{J}} / E_{\mathrm{C}}\right)$ at criticality $\left(R_{\mathrm{S}}=R_{\mathrm{Q}}\right)$. Moreover, the effective lead-to-grain resistance is within error-bars independent of $r$ and $R$, and depends only on $E_{\mathrm{J}} / E_{\mathrm{C}}$.

Given the success of the RG analysis of the two-junction model, we tried to use it in order to account for the above observations. Using simple scaling arguments, we extended the RG of [1] and section 2 to compute the effective lead-to-grain resistance at the critical point. This calculation also yielded a nearly $r$-independent effective resistance, and since in the limit $r=0, R=R_{\mathrm{Q}}$, the two-junction system and the single-junction system coincide, the observations about the resemblance of the two systems seem to be 
satisfactorily explained. The approximate RG-based resistance calculation fitted the QMC results quite well (figure 17).

But the observed similarity of the single- and two-junction systems could also be qualitatively interpreted in a more interesting way. The observation can be taken to indicate that, at criticality, each of the two junctions in the system sees an effective environment that imitates an $R_{\mathrm{s}}=R_{\mathrm{Q}}$ shunt, and therefore shows the same effective fixed-point resistance and critical properties as a single isolated junction at criticality. This mean-field argument was developed in section 6, and it allowed us to predict the location of the FSC-NOR phase boundary at intermediate Josephson energy well within error bars (figure 23). In addition, we used the RG predictions to compute the effective shunt resistance for each junction, and we found an astonishing result: in most of the ICFP region, the effective shunt for each junction does not deviate more than $1 \%$ from $R_{\mathrm{Q}}$ (figure 25 ).

The apparent success of a mean-field theory in this interacting system seems quite remarkable. Even more so is the fact that the RG-based calculation of the effective shunt on the critical manifold also yields with good accuracy $R_{\mathrm{Q}}$. This is indirect evidence for an internal structure in the coupled sine-Gordon theory that describes the two-junction system. If this approximation turns out to be generally valid, it may also give insight into the solution of coupled sine-Gordon models at intermediate couplings where no expansions can be done and little is analytically known.

The relevance of our work goes well beyond the two junction system. A closely related class of systems consists of resistively shunted Josephson junction arrays of two or more dimensions (see e.g. [7]). The RG equations derived for the two-junction system [1] were shortly thereafter also derived for a two-dimensional triangular array of resistively shunted Josephson junctions in the weak coupling regime: in [13], Tewari et al show that the weakly coupled triangular array undergoes a superconductor-to-metal transition almost identical to the direct NOR-FSC transition of the two-junction system (the RG equations for the two systems differ by one coefficient). Recall that this transition is in the interesting and novel ICFP region. Tewari et al also considered the square lattice, in which the RG equation are third order in the Josephson coupling, a difference which is unimportant near the ICFP. Hence, the work presented in this paper also verifies many of the results in [13].

In the future, we plan to adapt the Monte Carlo algorithm such that it can be applied to sizable arrays of Josephson junctions. This would allow for the first time direct controlled investigation of the Kosterlitz-Thouless NOR-SC* (normal-superconducting) transition in one-dimensional resistively shunted and free Josephson-junction arrays. Another interesting application for the algorithm would be Josephson-junction systems coupled to quasi-particle dissipation [17].

\section{Acknowledgments}

We acknowledge support by the Swiss National Science Foundation and NSF grant PHY99-07949, as well as helpful discussions with S Chakravarty, E Demler and D Fisher. Part of this work was completed at the Kavli Institute for Theoretical Physics, UCSB. We are also grateful for the hospitality of the Aspen Institute of Physics. The calculations 
have been performed on the Asgard and Hreidar Beowulf clusters at ETH Zürich, using the ALPS library [18].

\section{Appendix A: Critical exponents of the ICFP}

The behaviour of the two-junction system in the ICFP region at intermediate energies is determined by the critical properties of the unstable fixed point. Here we derive the critical exponents and principal directions of the RG in the weak and strong coupling regimes.

\section{A.1 Weak coupling}

The weak coupling RG equations are given by

$$
\begin{aligned}
& \frac{\mathrm{d} J}{\mathrm{~d} l}=-J u+\frac{R}{R_{\mathrm{Q}}} J J_{+}, \\
& \frac{\mathrm{d} J_{+}}{\mathrm{d} l}=-J_{+} w+\frac{r}{R_{\mathrm{Q}}} J^{2},
\end{aligned}
$$

where

$$
u=\frac{R+r}{R_{\mathrm{Q}}}-1, \quad w=\frac{2 R}{R_{\mathrm{Q}}}-1 .
$$

Near the unstable fixed point,

$$
J^{*}=\frac{R_{\mathrm{Q}}}{\sqrt{r R}} \sqrt{u w}, \quad J_{+}^{*}=\frac{R_{\mathrm{Q}}}{R} u,
$$

we can linearize equations (A.1) and (A.2) by writing

$$
J=J^{*}+j, \quad J_{+}=J_{+}^{*}+j_{+},
$$

and thus obtain

$$
\frac{\mathrm{d}}{\mathrm{d} l}\left(\begin{array}{c}
j \\
j_{+}
\end{array}\right)=\left(\begin{array}{cc}
0 & \sqrt{\frac{R}{r}} \sqrt{w u} \\
2 \sqrt{w u} \sqrt{\frac{r}{R}} & -w
\end{array}\right)\left(\begin{array}{c}
j \\
j_{+}
\end{array}\right) .
$$

The eigenvalues and eigenvectors of equation (A.6) give the relevant direction and exponent, and also the irrelevant direction and its decay. For the relevant direction one finds

$$
\begin{aligned}
& \lambda_{+}=\frac{1}{2}\left(-w+\sqrt{w^{2}+8 u w}\right), \\
& \left(j, j_{+}\right)=\left(1, \frac{1}{2} \sqrt{\frac{r}{R}}\left(-\sqrt{\frac{w}{u}}+\sqrt{\frac{w}{u}+8}\right)\right),
\end{aligned}
$$

and for the irrelevant direction

$$
\begin{aligned}
& \lambda_{-}=\frac{1}{2}\left(-w-\sqrt{w^{2}+8 u w}\right), \\
& \left(j, j_{+}\right)=\left(1, \frac{1}{2} \sqrt{\frac{r}{R}}\left(-\sqrt{\frac{w}{u}}-\sqrt{\frac{w}{u}+8}\right)\right) .
\end{aligned}
$$


Near the unstable fixed point we expect

$$
j \sim a T^{-\lambda_{+}}+b T^{-\lambda_{-}}, \quad j_{+} \sim a_{+} T^{-\lambda_{+}}+b_{+} T^{-\lambda_{-}},
$$

where $a$ and $b$ are determined by the initial $J^{(0)}$ and $J_{+}^{(0)}$. At very low $T$ we are close to the $J=J_{+}=0$ fixed point and have

$$
J \sim T^{u}, \quad J_{+} \sim T^{w} .
$$

If the system is superconducting, which means that $J^{(0)}$ and $J_{+}^{(0)}$ are in the region in which the couplings grow, then equation (A.11) still holds for intermediate temperatures, but as soon as the $J$ are far from the ICFP, the system crosses over to the strong coupling regime, where

$$
\zeta \sim T^{\left(R_{\mathrm{Q}}(R+r) /\left(2 R r+R^{2}\right)\right)-1},
$$

as follows from equation (17).

\section{A.2 Strong coupling regime}

The RG equations for the strong coupling case are

$$
\begin{aligned}
& \frac{\mathrm{d} \zeta}{\mathrm{d} l}=-\zeta \bar{u}+\frac{R R_{\mathrm{Q}}}{R^{2}+2 R r} \zeta \zeta_{\mathrm{D}}, \\
& \frac{\mathrm{d} \zeta_{\mathrm{D}}}{\mathrm{d} l}=-\zeta_{\mathrm{D}} \bar{v}+\frac{r R_{\mathrm{Q}}}{R^{2}+2 R r} \zeta^{2},
\end{aligned}
$$

where

$$
\bar{u}=\frac{R_{\mathrm{Q}}(R+r)}{2 R r+R^{2}}-1, \quad \bar{w}=\frac{2 R R_{\mathrm{Q}}}{R^{2}+2 R r}-1 .
$$

As in the weak coupling case we linearize these equations near the unstable fixed point

$$
\zeta^{*}=\frac{R^{2}+2 r R}{R_{\mathrm{Q}} \sqrt{r R}} \sqrt{\overline{u w}}, \quad \zeta_{\mathrm{D}}^{*}=\frac{R^{2}+2 r R}{R_{\mathrm{Q}} R} \bar{u},
$$

writing

$$
\zeta=\zeta^{*}+z, \quad \zeta_{\mathrm{D}}=\zeta_{\mathrm{D}}^{*}+z_{\mathrm{D}},
$$

and thus obtain

$$
\frac{\mathrm{d}}{\mathrm{d} l}\left(\begin{array}{c}
z \\
z_{\mathrm{D}}
\end{array}\right)=\left(\begin{array}{cc}
0 & \sqrt{\frac{R}{r}} \sqrt{\overline{w u}} \\
2 \sqrt{\overline{w u}} \sqrt{\frac{r}{R}} & -\bar{w}
\end{array}\right)\left(\begin{array}{c}
z \\
z_{\mathrm{D}}
\end{array}\right) .
$$

Equation (A.19) is essentially identical to equation (A.6), for the weak coupling case, except for the change of $u, w$ to $\bar{w}, \bar{u}$. Therefore all other results can be copied from the previous section as well. The eigenvalues and eigenvectors of the matrix in equation (A.19) are

$$
\begin{aligned}
& \lambda_{+}=\frac{1}{2}\left(-\bar{w}+\sqrt{\bar{w}^{2}+8 \overline{u w}}\right), \\
& \left(z, z_{\mathrm{D}}\right)=\left(1, \frac{1}{2} \sqrt{\frac{r}{R}}\left(-\sqrt{\frac{\bar{w}}{\bar{u}}}+\sqrt{\frac{\bar{w}}{\bar{u}}+8}\right)\right),
\end{aligned}
$$


and for the irrelevant direction

$$
\begin{aligned}
& \lambda_{-}=\frac{1}{2}\left(-\bar{w}-\sqrt{\bar{w}^{2}+8 \overline{u w}}\right), \\
& \left(z, z_{\mathrm{D}}\right)=\left(1, \frac{1}{2} \sqrt{\frac{r}{R}}\left(-\sqrt{\frac{\bar{w}}{\bar{u}}}-\sqrt{\frac{\bar{w}}{\bar{u}}+8}\right)\right) .
\end{aligned}
$$

Near the unstable fixed point we expect

$$
z \sim a T^{-\lambda_{+}}+b T^{-\lambda_{-}}, \quad z_{\mathrm{D}} \sim a_{+} T^{-\lambda_{+}}+b_{+} T^{-\lambda_{-}},
$$

just as for $j$ and $j_{+}$in equation (A.11) for the weak coupling case.

\section{Appendix B: Approximate calculation of the NOR-FSC phase boundary}

By using the result of appendix A for the ICFP and the critical exponents and irrelevant directions, equations (A.22) and (A.23), we can find an approximate implicit equation which can be solved for the critical $R$ as a function of $r$ and $\zeta_{0}$ (or $J_{0}$ ). The calculation is identical for the two limits; we will demonstrate it for the strong coupling limit.

The calculation is based on the simple assumption that for $r, R$ and $\zeta_{0}$ tuned to the critical values, the critical RG flow line between the point $\left(\zeta=\zeta_{0}, \zeta_{\mathrm{D}}=0\right)$ and the fixed point $\left(\zeta^{*}, \zeta_{\mathrm{D}}^{*}\right)$ given in equation $(27)$ is a straight line. This is illustrated in figure B.1. The slope of the line representing the critical manifold is

$$
\tan \theta=\left|z_{\mathrm{D}} / z\right|=\frac{1}{2} \sqrt{\frac{r}{R}}\left(\sqrt{\frac{\bar{w}}{\bar{u}}}+\sqrt{\frac{\overline{\bar{w}}}{\bar{u}}+8}\right),
$$

where $\left(z, z_{\mathrm{D}}\right)$ is defined in equation (A.23), and with $\bar{u}$ and $\bar{w}$ (which are functions of $r$ and $R$ ) given in equation (A.16). The implicit equation for $R_{\mathrm{c}}$ is

$$
\zeta_{\mathrm{D}} *=\left(\zeta_{0}-\zeta^{*}\right) \tan \theta .
$$

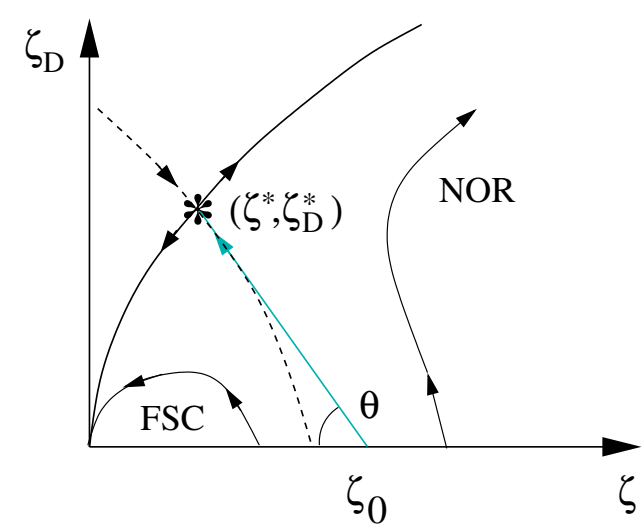

Figure B.1. We calculate the critical $R$ as a function of $r$ and $\zeta_{0}$ by assuming that the flow from the initial state $\left(\zeta_{0}, 0\right)$ to the fixed point $\left(\zeta^{*}, \zeta_{\mathrm{D}}^{*}\right)$ is a straight line. This turns out to be a reliable approximation throughout the ICFP region. 
More explicitly it becomes

$$
\frac{R^{2}+2 r R}{R_{\mathrm{Q}} R} \bar{u}=\left(\zeta_{0}-\frac{R^{2}+2 r R}{R_{\mathrm{Q}} \sqrt{r R}} \sqrt{\overline{u w}}\right) \frac{1}{2} \sqrt{\frac{r}{R}}\left(-\sqrt{\frac{\bar{w}}{\bar{u}}}-\sqrt{\frac{\bar{w}}{\bar{u}}+8}\right) .
$$

\section{Appendix C: Solution of the circuit in figure 21}

The effective lead-to-grain resistance is obtained by solving the circuit in figure 21 while replacing the ohmmeter with a current source providing a current $I$. The following equations express zero potential drop along the two loops in the circuit:

$$
\begin{aligned}
& \left(I+I_{1}\right) R+\left(I+I_{1}-I_{2}\right) r+I_{1} \alpha \zeta^{2}+\left(I_{1}-I_{2}\right) \alpha \zeta_{\mathrm{D}}^{2}=0, \\
& I_{2} R+I_{2} \alpha \zeta^{2}-\left(I_{1}-I_{2}\right) \alpha \zeta_{\mathrm{D}}^{2}=\left(I+I_{1}-I_{2}\right) .
\end{aligned}
$$

The solution for $I_{1}$ and $I_{2}$ is

$$
\begin{aligned}
& I_{1}=-I \cdot \frac{r\left(2 R+\alpha \zeta^{2}\right)+R\left(R+\alpha \zeta^{2}+\alpha \zeta_{\mathrm{D}}^{2}\right)}{\left(R+\alpha \zeta^{2}\right)\left(2 r+R+\alpha \zeta^{2}+2 \alpha \zeta_{\mathrm{D}}^{2}\right)}, \\
& I_{2}=I \cdot \frac{r \alpha \zeta^{2}-R \alpha \zeta_{\mathrm{D}}^{2}}{\left(R+\alpha \zeta^{2}\right)\left(2 r+R+\alpha \zeta^{2}+2 \alpha \zeta_{\mathrm{D}}^{2}\right)},
\end{aligned}
$$

and the potential drop on the junction connecting the grain $B$ and the lead $C$ is

$$
V_{\mathrm{BC}}=-\left(I_{1} \alpha \zeta^{2}+\left(I_{1}-I_{2}\right) \alpha \zeta_{\mathrm{D}}^{2}\right) .
$$

Substituting equations (C.3) and (C.4) into (C.5), and dividing by $I$, we obtain the effective resistance measured in the Monte Carlo calculations:

$$
R_{\mathrm{eff}}=\frac{\left(R^{2}+2 r R\right)\left(\zeta^{2}+\zeta_{\mathrm{D}}^{2}\right)+(R+r) \alpha \zeta^{2}\left(\zeta^{2}+2 \zeta_{\mathrm{D}}^{2}\right)}{\left(R / \alpha+\zeta^{2}\right)\left(2 r+R+\alpha \zeta^{2}+2 \alpha \zeta_{\mathrm{D}}^{2}\right)} .
$$

\section{References}

[1] Refael G et al, 2003 Phys. Rev. B 68214515

[2] Chakravarty S, 1982 Phys. Rev. Lett. 49681

Fröhlich J and Spencer T, 1982 Commun. Math. Phys. 8487

[3] Bulgadaev S A, 1984 Sov. Phys. JETP Lett. 39315

[4] Guinea F et al, 1985 Phys. Rev. Lett. 54263

[5] Fisher M P A and Zwerger W, 1985 Phys. Rev. B 326190

[6] Schmid A, 1983 Phys. Rev. Lett. 511506

[7] Chakravarty S et al, 1986 Phys. Rev. Lett. 562303

Chakravarty S et al, 1988 Phys. Rev. B 373283

[8] Panyukov S V and Zaikin A D, 1987 Phys. Lett. A 124325

Zwerger W, 1988 Physica B 152236

[9] Korshunov S E, 1989 Zh. Eksp. Teor. Fiz. 951058

Korshunov S E, 1989 Europhys. Lett. 9107

[10] Chow E, Delsing P and Haviland D B, 1998 Phys. Rev. Lett. 81204

[11] Takahide Y, Kanda A, Ootuka Y and Kobayashi S-I, 2000 Phys. Rev. Lett. 851974

[12] Refael G et al, 2005 Preprint cond-mat/0511212

[13] Tewari S et al, 2004 Preprint cond-mat/0407308

Tewari S et al, 2005 Preprint cond-mat/0501219 
[14] Werner P and Troyer M, 2005 Phys. Rev. Lett. 95060201

[15] Caldeira A O and Leggett A J, 1983 Ann. Phys., NY 149374

[16] Schön G and Zaikin A D, 1990 Phys. Rep. 198237

[17] Ambegaokar V, Eckern U and Schön G, 1982 Phys. Rev. Lett. 481745

[18] Troyer M et al, 1998 Lecture Notes in Computer Science vol 1505 (Berlin: Springer) p 191 Alet F et al, 2005 J. Phys. Soc. Japan. Suppl. 7430

Source codes of the library are available from http://alps.comp-phys.org/ 